\begin{tabular}{|c|c|c|c|c|c|}
\hline 4003 Fried LF & 2005 J Am Soc Nephrol & USA & 1 & 0 qua 4 vs 1 & 1.69 \\
\hline 4004 Messer LC & 2005 Matern Child Health J & USA & 1 & 1 qua 4 vs 1 & 3.4 \\
\hline 4006 Butler LM & 2005 Am J Respir Crit Care Med & USA & 1 & 0 qua 4 vs 1 & 1.43 \\
\hline 4008 Cocco P & 2005 Cancer Res & Italy & 0 & 1 qua 4 vs 1 & 2.00 \\
\hline 4010 Li G & 2005 Neurology & USA & 0 & 1 qua 4 vs 1 & 1.16 \\
\hline 4013 Larsson SC & 2005 Am J Clin Nutr & Sweden & 1 & 0 qua 4 vs 1 & 0.59 \\
\hline 4016 Beddhu S & 2005 J Ren Nutr & USA & 1 & 0 qua 4 vs 1 & 1.89 \\
\hline 4019 Thorand B & 2005 Diabetes & Germany & 1 & 0 qua 4 vs 1 & 1.73 \\
\hline 4020 Luchtenborg M & 2005 Cancer Causes Control & Netherlands & 1 & 1 qua 4 vs 1 & 1.61 \\
\hline 4021 Park HA & 2005 Atherosclerosis & USA & 1 & 1 qua 4 vs 1 & 1.99 \\
\hline 4022 Koushik A & $\begin{array}{c}2005 \text { Cancer Epidemiol } \\
\text { Biomarkers Prev }\end{array}$ & USA & 0 & 1 qua 4 vs 1 & 1.06 \\
\hline 4023 Rock CL & $2005 \mathrm{~J}$ Clin Oncol & USA & 1 & 0 qua 4 vs 1 & 0.57 \\
\hline 4025 Sornay-Rendu E & 2005 J Bone Miner Res & France & 1 & 0 qua 4 vs 1 & 2.20 \\
\hline 4030 Wakai K & 2005 Cancer Sci & Japan & 1 & 1 qua 4 vs 1 & 0.80 \\
\hline 4031 Ito $Y$ & $2005 \mathrm{~J}$ Epidemiol & Japan & 1 & 0 qua 4 vs 1 & 0.41 \\
\hline 4034 Merkin SS & 2005 Am J Kidney Dis & USA & 1 & 0 qua 4 vs 1 & 1.60 \\
\hline 4036 Schulze MB & 2005 Diabet Med & USA & 1 & 0 qua 4 vs 1 & 5.73 \\
\hline 4037 Bower M & 2005 Ann Intern Med & UK & 1 & 0 qua 4 vs 1 & 0.15 \\
\hline 4038 Whittemore AS & $2005 \mathrm{~J} \mathrm{Urol}$ & USA & 1 & 0 qua 4 vs 1 & 4.40 \\
\hline 4040 Rajpathak & 2005 J Am Coll Nut & USA & 1 & 1 qua 4 vs 1 & 0.43 \\
\hline
\end{tabular}

\begin{tabular}{|c|c|}
\hline 1 cystatin $C$ & $\begin{array}{l}\text { noncardiovascular } \\
\text { mortality }\end{array}$ \\
\hline 1 pregnancy intendness & $\begin{array}{l}\text { levels of perceived } \\
\text { stress during } \\
\text { pregnancy }\end{array}$ \\
\hline 1 meat-dim sum pattern & $\begin{array}{l}\text { new-onset cough with } \\
\text { phlegm }\end{array}$ \\
\hline 0 dichlorodiphenyltrichloroethane & $\begin{array}{l}\text { stomach cancer } \\
\text { mortality } \\
\text { dementia }\end{array}$ \\
\hline 1 high-fat dairy foods/d & colorectal cancer \\
\hline 1 total protein intake & low serum albumin \\
\hline 1 IL-18 & type 2 diabetes \\
\hline 0 Meat product consumption & $\begin{array}{l}\text { colon tumours with } \\
\text { truncating APC } \\
\text { mutation }\end{array}$ \\
\hline 1 saturated fat intake & coronary calcification \\
\hline 0 fruit intake & ovarian cancer \\
\hline $\begin{array}{l}1 \text { plasma total carotenoid } \\
\text { concentration } \\
1 \text { bone alkaline phosphatase }\end{array}$ & $\begin{array}{l}\text { new breast cancer } \\
\text { event } \\
\text { fracture }\end{array}$ \\
\hline 0 total fat intake & breast cancer \\
\hline 1 serum alpha-carotenes & lung cancer death \\
\hline $\begin{array}{l}1 \text { Individual-level socioeconomic } \\
\text { status area } \\
1 \text { proinsulin }\end{array}$ & $\begin{array}{l}\text { progressive Chronic } \\
\text { kidnety disease } \\
\text { diabetes }\end{array}$ \\
\hline $\begin{array}{l}1 \text { International Prognostic Index } \\
\text { (factors: age. tumor stage. } \\
\text { lactate dehydrogenase level. } \\
\text { Eastern Cooperative Oncology } \\
\text { Group performance status. and } \\
\text { number of extranodal sites) }\end{array}$ & death \\
\hline $\begin{array}{l}1 \text { serum Prostate specific } \\
\text { antigen } \\
1 \text { toenail selenium }\end{array}$ & prostate cancer \\
\hline
\end{tabular}




\begin{tabular}{|c|c|c|c|c|c|c|c|}
\hline 4041 Bergelt C & 2005 Cancer & Denmark & 1 & 0 qua 4 vs 1 & 0.80 & 1 vital exhaustion scores & cancer at all sites \\
\hline 4043 Schoen RE & 2005 Gastroenterology & USA & 1 & 1 qua 4 vs 1 & 1.70 & 1 IGF-I & adenoma \\
\hline 4047 Lukanova A & 2005 Int J Cancer & USA & 1 & 1 qua 4 vs 1 & 1.29 & $1 \mathrm{BMI}$ & any malignancy \\
\hline 4048 Mannisto S & 2005 Cancer Causes Control & Finland & 1 & 0 qua 4 vs 1 & 0.69 & $\begin{array}{l}1 \text { Pork, processed Meat, } \\
\text { potatoes }\end{array}$ & breast cancer \\
\hline 4050 Selvin E & 2005 Diabetes Care & USA & 1 & 0 qua 4 vs 1 & 2.46 & $1 \mathrm{HbA} 1 \mathrm{C}$ & $\begin{array}{l}\text { carotid intima-media } \\
\text { thickness }\end{array}$ \\
\hline $\begin{array}{l}4055 \text { Schernhammer } \\
\text { ES }\end{array}$ & 2005 J Natl Cancer Inst & USA & 1 & 1 qua 4 vs 1 & 0.59 & 16 -sulphatoxymelatonin & breast cancer \\
\hline 4056 Bergink AP & 2005 Bone & Netherlands & 1 & 0 qua 4 vs 1 & 2.80 & $\begin{array}{l}1 \text { femoral neck bone mineral } \\
\text { density }\end{array}$ & $\begin{array}{l}\text { radiographic } \\
\text { osteoarthritis of the } \\
\text { knee }\end{array}$ \\
\hline 4057 Leosdottir M & $2005 \mathrm{~J}$ Intern Med & Sweden & 1 & 0 qua 4 vs 1 & 1.46 & 1 total fat intake & cancer mortality \\
\hline 4058 Tsimikas S & 2005 N Engl J Med & USA & 1 & 0 qua 4 vs 1 & 3.12 & $\begin{array}{l}1 \text { oxidized phospholipid:apo B- } \\
100 \text { ratio }\end{array}$ & $\begin{array}{l}\text { coronary artery } \\
\text { disease }\end{array}$ \\
\hline 4059 Benner JS & 2005 Am J Health Syst Pharm & USA & 1 & 0 qua 4 vs 1 & 1.15 & $1 \mathrm{LDL}$ cholesterol reduction & $\begin{array}{l}\text { adherence to lipid- } \\
\text { lowering drug therapy. }\end{array}$ \\
\hline 4060 Wang J & 2005 Psychol Med & Canada & 1 & $\begin{array}{l}0 \text { qua } 4 \text { vs } \\
1+2+3\end{array}$ & 2.35 & 1 work stress & $\begin{array}{l}\text { major depressive } \\
\text { episodes }\end{array}$ \\
\hline 4062 Elkind MS. & 2005 Neurology & USA & 1 & 0 qua 4 vs 1 & 1.75 & 1 leukocyte count & ischemic stroke \\
\hline 4063 Ness AR & 2005 Heart & UK & 1 & 0 qua 4 vs 1 & 0.40 & 1 childhood intake of vegetables & stroke \\
\hline 4064 Seddon JM & 2005 Arch Ophthalmol & USA & 1 & 1 qua 4 vs 1 & 2.10 & $1 \mathrm{CRP}$ & $\begin{array}{l}\text { progression of age- } \\
\text { related macular } \\
\text { degeneration }\end{array}$ \\
\hline 4065 Silvera SA & 2005 Cancer Causes Control & USA & 0 & 1 qua 4 vs 1 & 1.43 & 0 glycemic index & pancreatic cancer \\
\hline 4066 Lundblad D & $2005 \mathrm{~J}$ Intern Med & Sweden & 1 & 0 qua 4 vs 1 & 19.1 & 1 tPA activity & myocardial infarction \\
\hline $4067 \operatorname{Lim}$ S & 2005 Diabetes Res Clin Pract & Korea & 1 & 0 qua 4 vs 1 & 2.36 & $1 \mathrm{CRP}$ & waist circumference \\
\hline 4068 Phipatanakul W & $\begin{array}{l}2005 \text { Ann Allergy Asthma } \\
\text { Immunol }\end{array}$ & USA & 1 & 0 qua 4 vs 1 & 2.32 & 1 levels of endotoxin & $\begin{array}{l}\text { wheeze in the first } \\
\text { year of life }\end{array}$ \\
\hline 4071 Van Guelpen B & 2005 Stroke & Sweden & 1 & 1 qua 4 vs 1 & 0.21 & 1 Plasma folate & hemorrhagic stroke \\
\hline 4073 Berndt SI. & 2005 Nutrition & USA & 0 & 1 qua 4 vs 1 & 1.31 & $\begin{array}{l}0 \text { plasma concentration of } \\
\text { vitamin } \mathrm{C}\end{array}$ & prostate cancer \\
\hline 4075 Yeh HC & 2005 Diabetes Care & USA & 1 & 0 qua 4 vs 1 & 1.60 & 1 Forced vital capacity & type 2 diabetes \\
\hline 4076 Sato $Y$ & 2005 Public Health Nutr & Japan & 0 & 1 qua 4 vs 1 & 1.13 & 0 fruit and vegetable intake & colon cancer \\
\hline 4077 Loucks EB & 2005 Psychosom Med & USA & 1 & 1 qua 4 vs 1 & 2.29 & 1 Social integration & $\begin{array}{l}\text { plasma concentrations } \\
\text { of fibrinogen }\end{array}$ \\
\hline 4079 Wattanakit K & 2005 Atherosclerosis & USA & 1 & 0 qua 4 vs 1 & 1.75 & 1 triglycerides & $\begin{array}{l}\text { peripheral arterial } \\
\text { disease }\end{array}$ \\
\hline 4081 Kaaks R & 2005 J Natl Cancer Inst & France & 1 & 1 qua 4 vs 1 & 1.73 & $\begin{array}{l}1 \text { serum concentrations of } \\
\text { testosterone }\end{array}$ & breast cancer \\
\hline
\end{tabular}




\begin{tabular}{|c|c|c|c|c|c|c|c|}
\hline 4082 Sesso HD & $\begin{array}{l}2005 \text { Cancer Epidemiol } \\
\text { Biomarkers Prev }\end{array}$ & USA & 0 & 1 qua 4 vs 1 & 0.93 & 0 plasma lycopene & breast cancer \\
\hline 4083 Nair GV & 2005 Chest & USA & 1 & 0 qua 4 vs 1 & 1.47 & 1 Pulse pressure & $\begin{array}{l}\text { Myocardial infarction } \\
\text { or Coronary heart } \\
\text { disease death }\end{array}$ \\
\hline 4085 Wong TY & 2005 Arch Intern Med & Australia & 1 & 0 qua 4 vs 1 & 1.53 & 1 arteriole-to-venule ratio & type 2 diabetes \\
\hline $\begin{array}{l}4088 \text { Garcia-Palmieri } \\
\text { MR }\end{array}$ & 2005 Nutr Metab Cardiovasc Dis & USA & 1 & 0 qua 4 vs 1 & 1.38 & 1 pulse pressure & $\begin{array}{l}\text { cardiovascular } \\
\text { mortality }\end{array}$ \\
\hline 4089 Wrensch M & 2005 Am J Epidemiol & USA & 1 & 0 qua 4 vs 1 & 0.41 & $\begin{array}{l}1 \text { immunoglobulin } \mathrm{G} \text { to varicella- } \\
\text { zoster virus }\end{array}$ & adult glioma \\
\hline 4091 Visser M & $\begin{array}{l}2005 \text { J Gerontol A Biol Sci Med } \\
\text { Sci }\end{array}$ & Netherlands & 1 & 0 qua 4 vs 1 & 1.90 & 1 muscle area & Mobility limitations \\
\hline 4092 Tsubono $\mathrm{Y}$ & $2005 \mathrm{Br} J$ Cancer & Japan & 0 & 1 qua 4 vs 1 & 0.92 & 0 fruit intake & colorectal cancer \\
\hline 4094 Mielke MM & 2005 Neurology & USA & 1 & 0 qua 4 vs 1 & 0.31 & 1 cholesterol levels & dementia \\
\hline 4095 Tworoger SS & 2005 J Natl Cancer Inst & USA & 1 & 1 qua 4 vs 1 & 1.70 & 0 free estradiol & breast cancer \\
\hline 4098 Svensson AM & 2005 Eur Heart J & Sweden & 1 & 0 qua 4 vs 1 & 2.66 & 1 blood glucose & all-cause mortality \\
\hline 4099 Lu M & 2005 Am J Clin Nutr & USA & 1 & 0 qua 4 vs 1 & 2.20 & 1 linolenic acid intake & Nuclear opacity \\
\hline 4101 Tsushima M & 2005 Dig Dis Sci & USA & 0 & 1 qua 4 vs 1 & 1.33 & 0 serum glucose & rectal cancer \\
\hline 4102 Tanne D & 2005 Stroke & Israel & 1 & 0 qua 4 vs 1 & 1.53 & 1 skinfold thickness & stroke mortality \\
\hline 4103 Stattin P & 2004 Cancer Causes Control & Sweden & 1 & 1 qua 4 vs 1 & 1.22 & 0 plasma enterolactone & prostate cancer \\
\hline 4104 De Cosmo S & 2005 Diabetes Care & Italy & 1 & 0 qua 4 vs 1 & 4.10 & $\begin{array}{l}1 \text { homeostasis model } \\
\text { assessment of insulin } \\
\text { resistance }\end{array}$ & $\begin{array}{l}\text { albumin-to-creatinine } \\
\text { ratio }\end{array}$ \\
\hline 4108 Mehling WE & 2005 J Psychosom Res & USA & 1 & 0 qua 4 vs 1 & 2.00 & 1 alexithymia summary scores & low back pain \\
\hline 4109 Margolis KL & 2005 Arch Intern Med & USA & 1 & 0 qua 4 vs 1 & 2.36 & 1 WBC counts & CHD death \\
\hline 4110 Meier C & 2005 J Bone Miner Res & Australia & 1 & 0 qua 4 vs 1 & 2.8 & $\begin{array}{l}1 \text { carboxyterminal cross-linked } \\
\text { telopeptide of type I collagen }\end{array}$ & fracture \\
\hline 4113 Al Snih S & 2004 Aging Clin Exp Res & USA & 1 & 0 qua 4 vs 1 & 1.90 & 1 hand grip strength & new ADL limitation \\
\hline 4114 Wang TJ & 2005 Circulation & USA & 1 & 0 qua 4 vs 1 & 1.93 & 1 urine albumin-creatinine ratio & hypertension \\
\hline 4115 Rumbold AR & $\begin{array}{l}2005 \text { Eur J Obstet Gynecol } \\
\text { Reprod Biol }\end{array}$ & Australia & 1 & $\begin{array}{l}0 \text { qua } 4 \text { vs } \\
1+2+3\end{array}$ & 1.75 & 1 vitamin $E$ intake & hypertensive disorders \\
\hline 4116 Hoffmeister A & 2005 Eur J Intern Med & Germany & 0 & 1 qua 4 vs 1 & 1.30 & $0 \mathrm{CRP}$ & cardiovascular event \\
\hline 4118 Hedblad B & 2005 Atherosclerosis & Sweden & 1 & 0 qua 4 vs 1 & 2.20 & $1 \mathrm{CO}$ exposure & CVD events \\
\hline 4120 Kojima M & 2005 Am J Epidemiol & Japan & 1 & 0 qua 4 vs 1 & 0.24 & $\begin{array}{l}1 \text { omega- } 3 \text { polyunsaturated fatty } \\
\text { acids }\end{array}$ & colorectal cancer \\
\hline 4121 Kragelund C & 2005 N Engl J Med & Denmark & 1 & 0 qua 4 vs 1 & 2.40 & 1 NT-pro-BNP levels & death from any cause \\
\hline 4123 Kurl S & 2005 Stroke & Finland & 1 & 0 qua 4 vs 1 & 2.70 & 1 exercise cardiac power & any stroke \\
\hline 4124 Kim MK & 2005 Int J Cancer & Japan & 1 & 1 qua 4 vs 1 & 2.06 & 1 traditional pattern & colon cancer \\
\hline
\end{tabular}




\begin{tabular}{|c|c|c|c|c|c|}
\hline 4126 Oei HH & 2005 Circulation & Netherlands & 1 & 1 qua 4 vs 1 & 1.97 \\
\hline 4129 Kado DM & 2005 Am J Med & USA & 1 & 0 qua 4 vs 1 & 1.60 \\
\hline 4132 Gilbert PB & $2005 \mathrm{~J}$ Infect Dis & USA & 1 & 1 qua 4 vs 1 & 0.22 \\
\hline 4136 Ray JG & 2005 Obstet Gynecol & Canada & 1 & 0 qua 4 vs 1 & 2.60 \\
\hline 4139 Bruno G & 2005 Diabetes Care & Italy & 1 & 1 qua 4 vs 1 & 1.20 \\
\hline 4140 Engstrom G & 2005 Diabetes & Sweden & 1 & 0 qua 4 vs 1 & 5.60 \\
\hline 4141 Montonen J & 2005 Eur J Clin Nutr & Finland & 1 & 0 qua 4 vs 1 & 0.69 \\
\hline 4142 Schupf N & 2005 J Am Geriatr Soc & USA & 1 & 0 qua 4 vs 1 & 1.80 \\
\hline 4145 Montonen J & 2005 Am J Epidemiol & Finland & 1 & 0 qua 4 vs 1 & 0.72 \\
\hline 4146 Taylor HA & 2005 Am J Cardiol & USA & 1 & 0 qua 4 vs 1 & 5.00 \\
\hline 4148 Mahabir S & $\begin{array}{l}2005 \text { Cancer Epidemiol } \\
\text { Biomarkers Prev }\end{array}$ & USA & 1 & 1 qua 4 vs 1 & 0.53 \\
\hline $\begin{array}{l}4149 \text { Raaschou- } \\
\text { Nielsen O }\end{array}$ & $\begin{array}{l}2005 \text { Cancer Epidemiol } \\
\text { Biomarkers Prev }\end{array}$ & Denmark & 1 & 1 qua 4 vs 1 & 0.70 \\
\hline 4150 Rinaldi S & $\begin{array}{c}2005 \text { Cancer Epidemiol } \\
\text { Biomarkers Prev }\end{array}$ & France & 1 & 1 qua 4 vs 1 & 1.93 \\
\hline 4151 Nakanishi N & 2005 Arch Intern Med & Japan & 1 & 0 qua 4 vs 1 & 0.54 \\
\hline 4153 Schulze MB & 2004 Diabetologia & USA & 1 & 0 qua 4 vs 1 & 1.97 \\
\hline 4154 Mai V & 2005 Int J Epidemiol & USA & 1 & 1 qua 4 vs 1 & 0.62 \\
\hline 4156 Thomson CC & $\begin{array}{l}2004 \text { J Public Health Manag } \\
\text { Pract }\end{array}$ & USA & 1 & 1 qua 4 vs 1 & 0.79 \\
\hline 4157 Tan ZS & 2005 Arch Neurol & USA & 1 & $\begin{array}{l}0 \text { qua } 4 \text { vs } \\
1+2+3\end{array}$ & 2.04 \\
\hline 4160 Shy BD & 2004 Prehosp Emerg Care & USA & 1 & $\begin{array}{l}0 \text { qua } 4 \text { vs } \\
1+2+3\end{array}$ & 0.42 \\
\hline 4161 van Gelder BM & 2004 Neurology & Netherlands & 1 & $\begin{array}{l}0 \text { qua } 4 \text { vs } \\
1+2+3\end{array}$ & 2.60 \\
\hline 4162 Engstrom G & 2004 J Vasc Surg & Sweden & 1 & 1 qua 4 vs 1 & 2.90 \\
\hline 4164 Schnabel R & 2005 Eur Heart J & Germany & 1 & 0 qua 4 vs 1 & 3.70 \\
\hline 4166 Pussinen PJ & $\begin{array}{l}2005 \text { Eur J Cardiovasc Prev } \\
\text { Rehabil }\end{array}$ & Finland & 1 & 1 qua 4 vs 1 & 3.99 \\
\hline
\end{tabular}

\begin{tabular}{|c|c|}
\hline 1 Lp-PLA2 activity & $\begin{array}{l}\text { coronary heart } \\
\text { disease }\end{array}$ \\
\hline 1 plasma folate & cognitive decline \\
\hline $\begin{array}{l}1 \text { antibody blocking of gp120 } \\
\text { binding to s-D4 } \\
1 \text { maternal weight }\end{array}$ & neural tube defects \\
\hline 1 fasting C-peptide & age \\
\hline 1 plasma proteins & Diabetes development \\
\hline 1 green vegetables & $\begin{array}{l}\text { type II diabetes } \\
\text { mellitus }\end{array}$ \\
\hline $\begin{array}{l}1 \text { total cholesterol. non-HDL-C. } \\
\text { and LDL-C } \\
1 \text { prudent pattern }\end{array}$ & $\begin{array}{l}\text { all cause mortality } \\
\text { type } 2 \text { diabetes }\end{array}$ \\
\hline $\begin{array}{l}1 \text { serum inflammatory markers } \\
\text { (fibrinogen and von Willebrand } \\
\text { Factor levels) } \\
1 \text { alcohol intake }\end{array}$ & $\begin{array}{l}\text { occurrence of } \\
\text { coronary heart } \\
\text { diseaseevents } \\
\text { renal cell cancer }\end{array}$ \\
\hline $\begin{array}{l}0 \text { 1.1-dichloro-2. 2-bis }(\mathrm{p}- \\
\text { chlorophenyl)ethylene } \\
\text { concentration }\end{array}$ & breast cancer \\
\hline $\begin{array}{l}1 \text { insulin-like growth factor-I } \\
\text { (IGF-I) }\end{array}$ & breast cancer \\
\hline 1 daily life energy expenditure & hypertension \\
\hline 1 non-HDL cholesterol & $\mathrm{CHD}$ \\
\hline $\begin{array}{l}1 \text { Recommended Foods Score } \\
\text { (RFS) }\end{array}$ & incident lung cancer \\
\hline 1 tax & experimentation \\
\hline 1 femoral neck BMD & $A D$ \\
\hline $\begin{array}{l}1 \text { time from patient collapse until } \\
\text { intubation }\end{array}$ & survival \\
\hline 1 activity duration & $\begin{array}{l}\text { 10-year cognitive } \\
\text { decline }\end{array}$ \\
\hline $\begin{array}{l}1 \text { inflammation-sensitive plasma } \\
\text { proteins (ISPs) }\end{array}$ & $\begin{array}{l}\text { peripheral artery } \\
\text { disease requiring } \\
\text { treatment }\end{array}$ \\
\hline $1 \mathrm{Nt}$-proBNP & cardiovascular risk \\
\hline
\end{tabular}




\begin{tabular}{|c|c|c|c|c|c|}
\hline 4167 De Michele M & $\begin{array}{l}2004 \text { Eur J Cardiovasc Prev } \\
\text { Rehabil }\end{array}$ & Italy & 1 & $\begin{array}{l}0 \text { qua } 4 \text { vs } \\
1+2+3\end{array}$ & 0.42 \\
\hline 4179 Baumgartner KB & 2004 Am J Epidemiol & USA & 1 & 0 qua 4 vs 1 & 0.16 \\
\hline 4181 Krause $N$ & 2004 Am J Ind Med & USA & 1 & 0 qua 4 vs 1 & 1.65 \\
\hline 4184 Chen C & 2005 Cancer & USA & 1 & 1 qua 4 vs 1 & 0.67 \\
\hline 4185 Esmaillzadeh A & 2005 Eur J Clin Nutr & Iran & 1 & 0 qua 4 vs 1 & 0.61 \\
\hline 4186 Suzuki K & $\begin{array}{c}2004 \text { Cancer Epidemiol } \\
\text { Biomarkers Prev }\end{array}$ & Japan & 1 & 1 qua 4 vs 1 & 3.40 \\
\hline 4187 Yuan JM & $\begin{array}{l}2004 \text { Cancer Epidemiol } \\
\text { Biomarkers Prev }\end{array}$ & USA & 1 & 1 qua 4 vs 1 & 0.38 \\
\hline 4189 Kitamura A & 2004 Stroke & Japan & 1 & 0 qua 4 vs 1 & 3.00 \\
\hline 4190 Giltay EJ & 2004 Arch Gen Psychiatry & Netherlands & 1 & 0 qua 4 vs 1 & 0.55 \\
\hline 4191 Sweeney C & 2004 Am J Epidemiol & USA & 1 & 1 qua 4 vs 1 & 1.44 \\
\hline 4192 Michaud DS & 2004 Am J Epidemiol & USA & 0 & 1 qua 4 vs 1 & 1.13 \\
\hline 4193 Rossi R & 2004 J Am Coll Cardiol & Italy & 1 & 0 qua 4 vs 1 & 5.77 \\
\hline 4194 Hill DJ & 2004 Pediatr Allergy Immunol & Australia & 1 & $\begin{array}{l}0 \text { qua } 4 \text { vs } \\
1+2+3\end{array}$ & 5.90 \\
\hline 4196 Luchsinger JA & 2004 Neurology & USA & 1 & 0 qua 4 vs 1 & 1.70 \\
\hline 4198 Dallaire F & 2004 Environ Health Perspect & Canada & 1 & 1 qua 4 vs 1 & 1.32 \\
\hline 4200 Vriesendorp TM & 2004 Eur J Vasc Endovasc Surg & Netherlands & 1 & 0 qua 4 vs 1 & 5.10 \\
\hline 4201 Kobayashi M & 2004 Nutr Cancer & Japan & 0 & 1 qua 4 vs 1 & 1.07 \\
\hline 4204 Nguyen L & $\begin{array}{l}2004 \text { J Acquir Immune Defic } \\
\text { Syndr }\end{array}$ & USA & 1 & $\begin{array}{l}0 \text { qua } 4 \text { vs } \\
1+2+3\end{array}$ & 3.92 \\
\hline 4207 Boekholdt SM & 2004 Am J Med & Netherlands & 1 & 0 qua 4 vs 1 & 1.60 \\
\hline 4028 Tworoger SS & 2004 Cancer Res & USA & 1 & 1 qua 4 vs 1 & 1.34 \\
\hline 4209 Ribeiro JC & 2004 Am J Hum Biol & Portugal & 1 & 0 qua 4 vs 1 & 2.00 \\
\hline 4210 Rusiecki JA & 2004 J Natl Cancer Inst & USA & 0 & 1 qua 4 vs 1 & 0.88 \\
\hline 4212 Elkins JS & 2004 Neurology & USA & 1 & 0 qua 4 vs 1 & 0.68 \\
\hline 4213 Bautista LE & 2005 J Hum Hypertens & USA & 1 & 0 qua 4 vs 1 & 2.30 \\
\hline 4215 Weinstein AR & 2004 JAMA & USA & 1 & 1 qua 4 vs 1 & 0.82 \\
\hline 4216 Manjer J & 2004 Int J Cancer & Sweden & 1 & 0 qua 4 vs 1 & 2.02 \\
\hline
\end{tabular}

\begin{tabular}{|c|c|}
\hline $1 \mathrm{HDL}-\mathrm{C}$ & $\begin{array}{l}\text { carotid intima-media } \\
\text { thickening }\end{array}$ \\
\hline 1 body mass index & tumor size \\
\hline $\begin{array}{l}1 \text { operators reporting ergonomic } \\
\text { problems }\end{array}$ & low back injury \\
\hline 0 IGF-I levels & $\begin{array}{l}\text { risk of prostate } \\
\text { carcinoma }\end{array}$ \\
\hline 1 whole-grain intake & hypertriglyceridemia \\
\hline 1 serum oxLDL & colorectal cancer \\
\hline 0 alpha-carotene & gastric cancer \\
\hline $\begin{array}{l}1 \text { maximum IMT of the common } \\
\text { carotid artery }\end{array}$ & stroke \\
\hline 1 optimism & all-cause mortality \\
\hline $1 \mathrm{BMI}$ & breast cancer \\
\hline 0 inorganic arsenic concentration & bladder cancer \\
\hline 1 flow-mediated dilation & hypertension \\
\hline 1 atopic dermatitis & $\begin{array}{l}\text { IgE-mediated food } \\
\text { allergy }\end{array}$ \\
\hline 1 insulin & Alzheimer disease \\
\hline 0 exposure to PCBs & $\begin{array}{l}\text { upper respiratory tract } \\
\text { infections }\end{array}$ \\
\hline 1 post-operative glucose levels & $\begin{array}{l}\text { post-operative } \\
\text { infections }\end{array}$ \\
\hline 0 fish consumption & colon cancer \\
\hline 1 HLA score & higher viral load \\
\hline $\begin{array}{l}1 \text { macrophage migration } \\
\text { inhibitory factor }\end{array}$ & $\begin{array}{l}\text { myocardial infarction } \\
\text { or death due to } \\
\text { coronary artery } \\
\text { disease }\end{array}$ \\
\hline 1 Prolactin & breast cancer \\
\hline $\begin{array}{l}1 \text { blood pressure. cholesterol. } \\
\text { and obesity } \\
0 \text { atrazine lifetime days }\end{array}$ & $\begin{array}{l}2 \text { or } 3 \text { risk factors of } \\
\text { metabolic syndrome } \\
\text { prostate cancer }\end{array}$ \\
\hline 1 Stroke risk & decline on the $3 \mathrm{MS}$ \\
\hline 1 interleukin 6 levels & High blood pressure \\
\hline 1 overall activity & diabetes \\
\hline 1 estrone & breast cancer \\
\hline
\end{tabular}




\begin{tabular}{|c|c|c|c|c|c|}
\hline 4217 English DR & $\begin{array}{c}2004 \text { Cancer Epidemiol } \\
\text { Biomarkers Prev }\end{array}$ & Australia & 1 & 1 qua 4 vs 1 & 2.30 \\
\hline 4219 Reijman M & 2004 Arthritis Rheum & Netherlands & 1 & 0 qua 4 vs 1 & 4.20 \\
\hline 4220 Dijk JM & 2004 Stroke & Netherlands & 1 & 0 qua 4 vs 1 & 2.10 \\
\hline 4221 Duncan BB & 2004 Diabetes & Brazil & 1 & 1 qua 4 vs 1 & 0.18 \\
\hline 4223 Saw SM & 2004 Invest Ophthalmol Vis Sci & Singapore & 1 & 0 qua 4 vs 1 & 2.40 \\
\hline 4229 Zhang C & 2004 Epidemiology & USA & 1 & 1 qua 4 vs 1 & 3.10 \\
\hline 4231 Kahn RS & 2004 Arch Pediatr Adolesc Med & USA & 1 & $\begin{array}{l}1 \text { qua } 4 \text { vs } \\
1+2+3\end{array}$ & 9.20 \\
\hline 4232 Stattin P & $2004 \mathrm{~J}$ Clin Oncol & Sweden & 1 & 1 qua 4 vs 1 & 1.67 \\
\hline 4234 Jiang $R$ & 2004 Diabetes Care & USA & 1 & 1 qua 4 vs 1 & 2.34 \\
\hline 4238 Rutter MK & 2004 Circulation & UK & 1 & 0 qua 4 vs 1 & 2.20 \\
\hline $\begin{array}{l}4241 \text { Gago-Dominguez } \\
\text { M }\end{array}$ & 2004 Carcinogenesis & USA & 1 & $\begin{array}{l}0 \text { qua } 4 \text { vs } \\
1+2+3\end{array}$ & 0.36 \\
\hline 4244 Arenillas JF & 2004 Neurology & Spain & 1 & 0 qua 4 vs 1 & 3.43 \\
\hline 4245 Sprenkle MD & 2004 Chest & USA & 1 & 0 qua 4 vs 1 & 5.47 \\
\hline 4249 Kant AK & 2004 J Nutr & USA & 1 & 1 qua 4 vs 1 & 0.72 \\
\hline 4250 Maru S & 2004 Am J Clin Nutr & Netherlands & 1 & 0 qua 4 vs 1 & 1.40 \\
\hline 4251 Okamura T & 2004 Am Heart J & Japan & 1 & 0 qua 4 vs 1 & 2.55 \\
\hline 4252 Hayashi T & 2004 Ann Intern Med & USA & 1 & 0 qua 4 vs 1 & 3.48 \\
\hline 4253 Skuladottir H & 2004 Lung Cancer & Denmark & 1 & 0 qua 4 vs 1 & 0.35 \\
\hline 4255 Luchsinger JA & 2004 Neurology & USA & 0 & 1 qua 4 vs 1 & 1.30 \\
\hline 4256 Navas-Acien A & 2004 Circulation & USA & 1 & 1 qua 4 vs 1 & 2.88 \\
\hline 4257 Page JH & $\begin{array}{l}2004 \text { Cancer Epidemiol } \\
\text { Biomarkers Prev }\end{array}$ & USA & 0 & 1 qua 4 vs 1 & 0.92 \\
\hline 4260 Boekholdt SM & $\begin{array}{l}2004 \text { Arterioscler Thromb Vasc } \\
\text { Biol }\end{array}$ & Netherlands & 1 & 0 qua 4 vs 1 & 1.72 \\
\hline 4261 Sheehan J. & $\begin{array}{l}2004 \text { Eur J Cardiovasc Prev } \\
\text { Rehabil }\end{array}$ & Ireland & 1 & 0 qua 4 vs 1 & 2.14 \\
\hline 4264 Oh JC & $\begin{array}{c}2004 \text { Cancer Epidemiol } \\
\text { Biomarkers Prev }\end{array}$ & USA & 1 & 0 qua 4 vs 1 & 9.67 \\
\hline
\end{tabular}

0 consumption of fresh red meat rectal cancer

$1 \mathrm{C}$-telopeptide fragments of radiographic $\mathrm{OA}$ of the type II collagen level knee

1 Arterial stiffness ischemic stroke or TIA

1 adiponectin levels

1 nonverbal IQ

diabetes

1 Plasma ascorbic acid

concentrations

1 parents mental health

myopia

gestational diabetes

high BPI-EXT score

1 plasma peptide levels of IGF-I prostate cancer

1 non-HDL cholesterol cardiovascular

\section{CRP levels}

1 marine $n-3$ fatty acids

$1 \mathrm{Lp}(\mathrm{a})$ lipoprotein levels$$
\text { disease }
$$

CVD events

post-menopausal

breast cancer

greater extent of

intracranial large-

artery occlusive

disease.

1 physical component summary death

1 Recommended Foods and

all-cause mortality

Behavior Score

$$
1 \mathrm{BMI}
$$

1 resting heart rate $(\mathrm{HR})$

1 intra-abdominal fat area

1 intake of plant food

0 homocysteine

0 Lead levels

0 DHEA

1 IL-8

1 corrected QT dispersion

1 IGF-2

\section{mortality from all}

causes

cardiovascular deaths

hypertension

lung cancer

Alzheimer disease

peripheral arterial

disease

breast cancer

future CAD

cardiac death

endometrial cancer 


\begin{tabular}{|c|c|c|c|c|c|}
\hline 4267 van Meurs JB & 2004 N Engl J Med & Netherlands & 1 & $\begin{array}{l}0 \text { qua } 4 \text { vs } \\
1+2+3\end{array}$ & 1.90 \\
\hline 4270 Zhang C & 2004 J Reprod Med & USA & 1 & 0 qua 4 vs 1 & 12.8 \\
\hline $4274 \operatorname{Lin} Y$ & 2004 Int J Cancer & Japan & 0 & 1 qua 4 vs 1 & 2.31 \\
\hline 4275 Butler LM & 2004 Am J Respir Crit Care Med & USA & 1 & 0 qua 4 vs 1 & 0.61 \\
\hline 4276 Gunnarsdottir I & 2004 Eur J Clin Nutr & Iceland & 1 & 0 qua 4 vs 1 & 0.70 \\
\hline 4278 Pelfrene E & 2004 Soc Sci Med & Belgium & 1 & 1 qua 4 vs 1 & 1.91 \\
\hline 4279 Wong TY & 2004 Am J Epidemiol & Australia & 0 & 1 qua 4 vs 1 & 0.93 \\
\hline 4280 Kim MK & 2004 Int J Cancer & Japan & 1 & 0 qua 4 vs 1 & 0.56 \\
\hline 4283 Blake GJ & 2004 Arch Intern Med & USA & 1 & $\begin{array}{l}1 \text { qua } 4 \text { vs } \\
1+2+3\end{array}$ & 2.25 \\
\hline 4287 Turner BJ & 2004 Ann Intern Med & USA & 1 & 0 qua 4 vs 1 & 0.79 \\
\hline 4288 Maclnnis RJ & $\begin{array}{l}2004 \text { Cancer Epidemiol } \\
\text { Biomarkers Prev }\end{array}$ & Australia & 1 & 0 qua 4 vs 1 & 2.30 \\
\hline 4294 Schulze MB & 2004 Diabetes Care & USA & 1 & 0 qua 4 vs 1 & 2.62 \\
\hline 4297 Anderson JP & 2004 Cancer & USA & 1 & 1 qua 4 vs 1 & 1.86 \\
\hline 4302 Morange PE & 2004 Circulation & France & 1 & 0 qua 4 vs 1 & 3.04 \\
\hline 4303 Horne BD & 2004 Ann Epidemiol & USA & 1 & 1 qua 4 vs 1 & 1.32 \\
\hline 4305 Samelson EJ & 2004 Am J Epidemiol & USA & 1 & 1 qua 4 vs 1 & 0.73 \\
\hline 4307 Larsson SC & 2004 J Natl Cancer Inst & Sweden & 1 & 1 qua 4 vs 1 & 0.67 \\
\hline 4308 Panichi V & 2004 Nephrol Dial Transplant & Italy & 1 & 1 qua 4 vs 1 & 5.20 \\
\hline 4311 Hubbard JS & 2004 Urology & USA & 0 & 1 qua 4 vs 1 & 2.06 \\
\hline 4313 Rosenthal AD & $\begin{array}{l}2004 \text { Int J Obes Relat Metab } \\
\text { Disord }\end{array}$ & USA & 1 & 0 qua 4 vs 1 & 2.57 \\
\hline 4315 Erlinger TP & 2004 JAMA & USA & 1 & 1 qua 4 vs 1 & 2.55 \\
\hline 4317 Keinan-Boker L & 2004 Am J Clin Nutr & Netherlands & 0 & 1 qua 4 vs 1 & 1.00 \\
\hline 4318 Massy ZA & $\begin{array}{l}2004 \text { Am J Physiol Renal } \\
\text { Physiol }\end{array}$ & France & 1 & $\begin{array}{l}0 \text { qua } 4 \text { vs } \\
1+2+3\end{array}$ & 3.30 \\
\hline 4319 Golden SH & 2004 Diabetes Care & USA & 1 & 0 qua 4 vs 1 & 1.63 \\
\hline 4320 Montonen J & 2004 Diabetes Care & Finland & 1 & 0 qua 4 vs 1 & 0.69 \\
\hline
\end{tabular}

1 homocysteine level
1 daily vitamin C intake
0 IGF-I levels
1 nonstarch polysaccharides
intake
1 birth weight

1 evel of job demands

0 retinal arteriole-to-venule ratio all-cause mortality

1 healthy pattern

$1 \mathrm{~A}(1 \mathrm{c})$ levels

1 physician visit adherence

1 fat-free mass (FFM)

1 CRP

1 height

1 plasma vWF levels

1 geographic Socio economic status measure-residential economic status

1 metacarpal cortical area

0 dietary folate intake

$1 \mathrm{IL}-6$

0 waist/hip ratio

1 body mass index

1 CRP

0 intakes of isoflavones

1 plasma S-nitrosothio concentrations

1 depressive symptoms

1 Vitamin E intake fracture

gestational diabetes mellitus

death from pancreatic

symptoms

ove the 90th percentile of truncal

gastric cancer

cardiovascular events

attending the first colon study male colon cancer

cardiovascular events

ovarian cancer

hard CHD

death/MI

coronary heart disease

ovarian cancer death from all causes

prostate cancer

diabetes

colon cancer

breast cancer

cardiac events

Diabetes development type 2 diabetes 


\begin{tabular}{|c|c|c|c|c|c|}
\hline 4321 Takebayashi T & 2004 Occup Environ Med & Japan & 1 & 0 qua 4 vs 1 & 3.90 \\
\hline 4329 Molyneux A & 2004 Am J Epidemiol & UK & 1 & 0 qua 4 vs 1 & 1.78 \\
\hline 4331 Church TS & 2004 Diabetes Care & USA & 1 & 1 qua 4 vs 1 & 4.50 \\
\hline 4332 Pankow JS & 2004 Diabetes Care & USA & 1 & 0 qua 4 vs 1 & 1.68 \\
\hline 4334 Wolf $\mathrm{M}$ & 2004 Diabetes Care & USA & 1 & 1 qua 4 vs 1 & 4.90 \\
\hline 4337 Wong TY & 2004 Diabetes & Australia & 1 & $\begin{array}{l}0 \text { qua } 4 \text { vs } \\
1+2+3\end{array}$ & 1.53 \\
\hline 4340 Engstrom G & $\begin{array}{l}2004 \text { Arterioscler Thromb Vasc } \\
\text { Biol }\end{array}$ & Sweden & 1 & 1 qua 4 vs 1 & 3.20 \\
\hline 4341 Sesso HD & 2004 Am J Clin Nutr & USA & 1 & 1 qua 4 vs 1 & 0.62 \\
\hline 4342 Rosengren A & 2004 Eur Heart J & Sweden & 1 & 0 qua 4 vs 1 & 0.45 \\
\hline 4351 Lukanova A & 2004 Int J Cancer & France & 1 & 1 qua 4 vs 1 & 4.13 \\
\hline $\begin{array}{l}4353 \text { Wannamethee } \\
\text { SG }\end{array}$ & 2004 Diabetologia & UK & 1 & 0 qua 4 vs 1 & 0.66 \\
\hline 4354 Kilkkinen A & 2004 Int J Cancer & Finland & 0 & 1 qua 4 vs 1 & 1.30 \\
\hline 4377 Guerra S & 2004 Am J Respir Crit Care Med & USA & 1 & $\begin{array}{l}0 \text { qua } 4 \text { vs } \\
1+2+3\end{array}$ & 4.50 \\
\hline 5001 Gelber RP & 2005 Am J Kidney Dis & USA & 1 & 0 qui 5 vs 1 & 1.45 \\
\hline 5006 Jee SH & 2004 Am J Epidemiol & Korea & 1 & 0 qui 5 vs 1 & 2.1 \\
\hline 5009 Song $Y$ & 2004 Am J Cardiol & USA & 0 & 1 qui 5 vs 1 & 0.87 \\
\hline 5010 Nothlings U & 2005 J Natl Cancer Inst & USA & 1 & 0 qui 5 vs 1 & 1.68 \\
\hline 5011 Wright ME & 2005 Cancer Causes Control & USA & 0 & 1 qui 5 vs 1 & 1.15 \\
\hline 5012 Selvin E & 2005 Arch Intern Med & USA & 1 & 1 qui 5 vs 1 & 2.37 \\
\hline 5013 Kurth T & 2005 Arch Intern Med & USA & 0 & 1 qui 5 vs 1 & 1.12 \\
\hline 5014 Zhang $X$ & 2005 Arch Intern Med & USA & 1 & 0 qui 5 vs 1 & 0.63 \\
\hline 5015 Schulze MB & 2005 Am J Clin Nutr & Germany & 1 & 0 qui 5 vs 1 & 3.09 \\
\hline
\end{tabular}

1 six year mean TTCA

1 smoking prevalence

1 fitness

1 free fatty acids

1 leukocyte count

1 retinal venular diameter

1 ISPs (fibrinogen. orosomucoid. alpha1-antitrypsin. haptoglobin. and ceruloplasmi

0 plasma lycopene

1 social integration

1 estradiol

1 birthweight SD scores

0 enterolactone

1 IFNgamma production at 3 months

$1 \mathrm{BMI}$

1 white blood cell count

0 magnesium intake

1 daily intake of processed meat

0 renal net acid excretion

1 Diabetic adults: $\mathrm{HbA}(1 \mathrm{c})$ level

0 analgesic use

1 soy protein intake

1 Diet pattern. high in sugarsweetened soft drinks. refined grains. diet soft drinks. and processed meat but low in wine. coffee. cruciferous vegetables. and yellow vegetables

0 vitamin $D$ intake

ischaemic findings incident smoking all-cause mortality type 2 diabetes

gestational diabetes mellitus

gross proteinuria aneurysms

Cardiovascula disease coronary morbidity endometrial cancer HOMA insulin resistance score breast cancer

developing recurrent wheezing chronic kidney disease atherosclerotic cardiovascula diseases Cardiovascular disease

tic cance bladder cancer

hypertension fracture diabetes

.

o




\begin{tabular}{|c|c|c|c|c|c|c|c|}
\hline 5020 Mommers M & 2005 Cancer & Netherlands & 1 & 1 qui 5 vs 1 & 0.98 & 0 total vegetables intake & ovarian carcinoma \\
\hline 5021 Zhang SM & $\begin{array}{l}2005 \text { Cancer Epidemiol } \\
\text { Biomarkers Prev }\end{array}$ & USA & 1 & 1 qui 5 vs 1 & 0.81 & 1 total folate intake & ER- tumors \\
\hline $\begin{array}{l}5022 \text { Bertone-Johnson } \\
\text { ER }\end{array}$ & $\begin{array}{l}2005 \text { Cancer Epidemiol } \\
\text { Biomarkers Prev }\end{array}$ & USA & 0 & 1 qui 5 vs 1 & 0.73 & $\begin{array}{l}0 \text { plasma levels of } 25- \\
\text { hydroxyvitamin } D[25(\mathrm{OH}) \mathrm{D}]\end{array}$ & breast cancer \\
\hline 5024 Batty GD & 2005 Br J Psychiatry & Denmark & 1 & 0 qui 5 vs 1 & 1.70 & 1 early-life IQ & $\begin{array}{l}\text { subsequent } \\
\text { psychiatric disorder in } \\
\text { adulthood }\end{array}$ \\
\hline 5025 Ridker PM & 2005 JAMA & USA & 1 & 0 qui 5 vs 1 & 1.62 & 1 LDL-C & $\begin{array}{l}\text { future cardiovascular } \\
\text { events }\end{array}$ \\
\hline 5029 Tsai CJ & 2005 Gastroenterology & USA & 1 & 0 qui 5 vs 1 & 1.35 & 1 dietary carbohydrate & cholecystectomy \\
\hline 5031 Myint PK & 2005 Ann Epidemiol & UK & 1 & 0 qui 5 vs 1 & 2.15 & 1 SF-36 scores & all cause mortality \\
\hline 5032 Mucci LA & 2005 Int J Cancer & USA & 0 & 1 qui 5 vs 1 & 0.90 & 0 acrylamide intake & colorectal cancer \\
\hline 5033 Shai I & 2005 Diabetologia & USA & 1 & 1 qui 5 vs 1 & 1.95 & 1 lipoprotein (Lp)(a) & $\mathrm{CHD}$ \\
\hline 5034 Mozaffarian D & 2005 J Am Coll Cardiol & USA & 1 & 1 qui 5 vs 1 & 0.73 & 1 long-chain n-3 fatty acid intake & $\mathrm{CHF}$ \\
\hline $\begin{array}{l}5037 \text { Bertone-Johnson } \\
\text { ER }\end{array}$ & 2005 Arch Intern Med & USA & 1 & 0 qui 5 vs 1 & 0.59 & 1 total vitamin $D$ intake & $\begin{array}{l}\text { Premenstrual } \\
\text { syndrome }\end{array}$ \\
\hline $5038 \operatorname{Lin} J$ & 2005 Cancer Causes Control & USA & 1 & 1 qui 5 vs 1 & 0.79 & 0 fruit intake & colorectal cancer \\
\hline $5039 \operatorname{Sin} \mathrm{DD}$ & 2005 Chest & Canada & 1 & 0 qui 5 vs 1 & 3.36 & 1 FEV1 & $\begin{array}{l}\text { ischemic heart } \\
\text { disease }\end{array}$ \\
\hline 5043 Larsson SC & 2005 Gastroenterology & Sweden & 1 & 0 qui 5 vs 1 & 0.66 & 1 vitamin B6 intake & colorectal cancer \\
\hline 5046 Sesso HD & $\begin{array}{l}2005 \text { Cancer Epidemiol } \\
\text { Biomarkers Prev }\end{array}$ & USA & 0 & 1 qui 5 vs 1 & 1.00 & 0 dietary lycopene & breast cancer \\
\hline 5048 Heidemann C & 2005 Diabetologia & Germany & 1 & 0 qui 5 vs 1 & 0.27 & 1 dietary pattern score & type 2 diabetes \\
\hline 5049 Tsai CJ & 2005 Gut & USA & 1 & 0 qui 5 vs 1 & 1.59 & 1 carbohydrate intake & $\begin{array}{l}\text { symptomatic gall } \\
\text { stone disease }\end{array}$ \\
\hline 5050 Tsai CJ & 2005 Arch Intern Med & USA & 1 & 1 qui 5 vs 1 & 1.23 & $\begin{array}{l}1 \text { dietary intake of trans-fatty } \\
\text { acids }\end{array}$ & gallstone disease \\
\hline 5051 Lu M & 2005 Am J Epidemiol & USA & 1 & 1 qui 5 vs 1 & 1.10 & 0 total fat intake & cataract extraction \\
\hline 5053 Vrbova L & 2005 J Negat Results Biomed & Canada & 0 & 1 qui 5 vs 1 & 1.03 & 0 income & death \\
\hline 5055 Slinin Y & 2005 J Am Soc Nephrol & USA & 1 & 0 qui 5 vs 1 & 1.08 & 1 calcium levels & cardiovascular events \\
\hline 5056 Michaud DS & 2005 J Natl Cancer Inst & USA & 0 & 1 qui 5 vs 1 & 1.32 & 0 prudent dietary pattern & pancreatic cancer \\
\hline 5060 Lin J & 2005 Am J Epidemiol & USA & 0 & 1 qui 5 vs 1 & 1.20 & 0 Intakes of total calcium & colorectal cancer \\
\hline 5063 Larsson SC & $\begin{array}{c}2005 \text { Cancer Epidemiol } \\
\text { Biomarkers Prev }\end{array}$ & Sweden & 1 & 1 qui 5 vs 1 & 0.61 & 1 dietary folate intake & colon cancer \\
\hline $\begin{array}{l}5064 \text { Schernhammer } \\
\text { ES }\end{array}$ & $\begin{array}{c}2005 \text { Cancer Epidemiol } \\
\text { Biomarkers Prev }\end{array}$ & USA & 1 & 1 qui 5 vs 1 & 1.00 & 0 IGF-I & breast cancer \\
\hline 5065 Fung TT & 2005 Int J Cancer & USA & 1 & 0 qui 5 vs 1 & 1.44 & 1 Western pattern score & breast cancer \\
\hline 5066 Frost L & 2005 Am J Clin Nutr & Denmark & 0 & 1 qui 5 vs 1 & 0.91 & 0 caffeine consumption & $\begin{array}{l}\text { atrial fibrillation or } \\
\text { flutter }\end{array}$ \\
\hline
\end{tabular}




\begin{tabular}{|c|c|c|c|c|c|c|c|}
\hline 5067 Wang $Y$ & 2005 Am J Clin Nutr & USA & 1 & 0 qui 5 vs 1 & 12.0 & 1 waist circumference & type 2 diabetes \\
\hline 5068 Alonso A & 2004 Lipids & Spain & 1 & 1 qui 5 vs 1 & 0.46 & 1 olive oil consumption & hypertension \\
\hline 5071 Madsen TE & 2005 Am J Nephrol & USA & 1 & $\begin{array}{l}0 \text { qui } 5 \text { vs } \\
1+2+3+4\end{array}$ & 1.90 & 1 model including NAF cytology & all-cause mortality \\
\hline 5074 Taylor EN & 2005 Am J Kidney Dis & USA & 1 & 0 qui 5 vs 1 & 1.28 & $\begin{array}{l}1 \text { docosahexaenoic acid } \\
\text { eicosapentaenoic acid }\end{array}$ & kidney stones \\
\hline 5076 Hartman TJ & 2005 J Nutr & USA & 1 & 1 qui 5 vs 1 & 0.91 & 0 dietary calcium intake & adenoma recurrence \\
\hline 5077 Michaud DS & $\begin{array}{l}2005 \text { Cancer Epidemiol } \\
\text { Biomarkers Prev }\end{array}$ & USA & 1 & 1 qui 5 vs 1 & 1.32 & 0 Dietary glycemic load & colorectal cancer \\
\hline 5078 Flood A & $\begin{array}{l}2005 \text { Cancer Epidemiol } \\
\text { Biomarkers Prev }\end{array}$ & USA & 1 & 1 qui 5 vs 1 & 0.74 & 1 dietary calcium & colorectal cancer \\
\hline 5080 Rouillier $P$ & 2005 Alcohol Clin Exp Res & France & 1 & 0 qui 5 vs 1 & 1.45 & 1 Alcohol intake & Abnormal ApoB \\
\hline 5081 van Gils $\mathrm{CH}$ & 2005 JAMA & Netherlands & 0 & 1 qui 5 vs 1 & 0.98 & 0 total vegetables & breast cancer \\
\hline 5082 Frost L & 2005 Am J Clin Nutr & Denmark & 1 & 0 qui 5 vs 1 & 1.44 & $\begin{array}{l}1 \text { n-3 fatty acids consumed from } \\
\text { fish }\end{array}$ & $\begin{array}{l}\text { atrial fibrillation or } \\
\text { flutter }\end{array}$ \\
\hline 5083 Larsson SC & 2005 JAMA & Sweden & 1 & 1 qui 5 vs 1 & 0.59 & 1 magnesium intake & colorectal cancer \\
\hline 5085 Silvera SA & 2005 Int J Cancer & USA & 1 & 1 qui 5 vs 1 & 0.78 & $\begin{array}{l}0 \text { postmenopausal women: } \\
\text { glycemic index }(\mathrm{GI})\end{array}$ & breast cancer \\
\hline 5086 Adebamowo CA & 2005 Int J Cancer & USA & 1 & 1 qui 5 vs 1 & 0.94 & 0 sum of flavonols intake & breast cancer \\
\hline 5087 Knekt P & 2004 Am J Clin Nutr & Finland & 1 & 1 qui 5 vs 1 & 0.84 & 0 dietary intake for vitamin $\mathrm{E}$ & $\begin{array}{l}\text { Coronary heart } \\
\text { disease }\end{array}$ \\
\hline 5088 Jensen MK & 2004 Am J Clin Nutr & USA & 1 & 0 qui 5 vs 1 & 0.82 & 1 whole-grain intake & $\begin{array}{l}\text { Coronary heart } \\
\text { disease }\end{array}$ \\
\hline 5089 Taylor EN & 2004 J Am Soc Nephrol & USA & 1 & 0 qui 5 vs 1 & 0.69 & 1 dietary calcium & kidney stones \\
\hline 5090 Wu K & 2004 Cancer Causes Control & USA & 1 & 1 qui 5 vs 1 & 0.84 & 0 prudent pattern scores & colon cancer \\
\hline 5092 Koenig W & 2005 Clin Chem & Germany & 1 & 0 qui 5 vs 1 & 2.27 & 1 cystatin $\mathrm{C}$ distribution & $\begin{array}{l}\text { secondary } \\
\text { Cerebrovascular } \\
\text { disease events }\end{array}$ \\
\hline 5093 Fung TT & 2004 Arch Intern Med & USA & 1 & 0 qui 5 vs 1 & 1.49 & 1 Western pattern & $\begin{array}{l}\text { type } 2 \text { diabetes in } \\
\text { women }\end{array}$ \\
\hline 5094 Khaw KT & 2004 Am J Clin Nutr & UK & 1 & 0 qui 5 vs 1 & 2.48 & 1 urinary sodium & $\begin{array}{l}\text { systolic blood } \\
\text { pressure }>/=160 \mathrm{~mm} \\
\mathrm{Hg}\end{array}$ \\
\hline 5095 Hung HC & 2004 J Natl Cancer Inst & USA & 1 & 1 qui 5 vs 1 & 0.95 & 0 total fruit and vegetable intake & major chronic disease \\
\hline 5096 Lin J & 2004 Am J Epidemiol & USA & 1 & 0 qui 5 vs 1 & 1.86 & 1 intake of fried foods & colorectal cancer \\
\hline 5097 Folsom AR & 2004 Am J Epidemiol & USA & 1 & 0 qui 5 vs 1 & 0.82 & 1 fish intake & total mortality \\
\hline 5098 Wolk R & 2004 J Am Coll Cardiol & USA & 1 & 0 qui 5 vs 1 & 6.46 & 1 leptin levels & $\begin{array}{l}\text { future cardiovascular } \\
\text { events }\end{array}$ \\
\hline 5099 Ford ES & 2004 Eur J Clin Nutr & USA & 1 & 1 qui 5 vs 1 & 0.68 & 1 grain consumption & $\begin{array}{l}\text { elevated C-reactive } \\
\text { protein }\end{array}$ \\
\hline
\end{tabular}




\begin{tabular}{|c|c|c|c|c|c|}
\hline 5100 Empana JP & 2004 Circulation & France & 1 & 0 qui 5 vs 1 & 2.60 \\
\hline 5101 Frost L & 2004 Arch Intern Med & Denmark & 1 & 1 qui 5 vs 1 & 1.46 \\
\hline 5103 Luo ZC & 2004 Epidemiology & Canada & 1 & 0 qui 5 vs 1 & 1.44 \\
\hline 5106 Tsai CJ & 2005 Ann Intern Med & USA & 1 & 0 qui 5 vs 1 & 0.82 \\
\hline 5108 Erkkila AT & 2005 Eur J Clin Nutr & USA & 1 & 1 qui 5 vs 1 & 0.79 \\
\hline 5113 Hutchings A & 2004 Med Care & UK & 1 & 0 qui 5 vs 1 & 0.70 \\
\hline 5117 Singh SM & 2004 CMAJ & Canada & 1 & 0 qui 5 vs 1 & 1.38 \\
\hline 5118 Boekholdt SM & 2004 Circulation & Netherlands & 1 & 0 qui 5 vs 1 & 1.43 \\
\hline 5119 Alonso A & 2004 Br J Nutr & Spain & 1 & 1 qui 5 vs 1 & 0.58 \\
\hline 5120 Smith W & 2004 Hypertension & Australia & 1 & 0 qui 5 vs 1 & 2.60 \\
\hline 5122 Chen H & 2004 Am J Epidemiol & USA & 0 & 1 qui 5 vs 1 & 1.00 \\
\hline 5124 Schaumberg DA & 2004 Am J Clin Nutr & USA & 0 & 1 qui 5 vs 1 & 0.95 \\
\hline 5125 Schulze MB & 2004 Am J Clin Nutr & USA & 1 & 1 qui 5 vs 1 & 1.59 \\
\hline 5127 Leung H & $2004 \mathrm{~J}$ Hypertens & Australia & 1 & 0 qui 5 vs 1 & 2.40 \\
\hline 5129 Oh K & $\begin{array}{c}2004 \text { Cancer Epidemiol } \\
\text { Biomarkers Prev }\end{array}$ & USA & 0 & 1 qui 5 vs 1 & 1.11 \\
\hline 5130 Cho E & 2004 J Natl Cancer Inst & USA & 1 & 1 qui 5 vs 1 & 0.86 \\
\hline 5131 Tsai CJ & 2004 Am J Gastroenterol & USA & 1 & 1 qui 5 vs 1 & 0.87 \\
\hline 5132 Fung TT & 2004 Stroke & USA & 1 & 1 qui 5 vs 1 & 1.58 \\
\hline 5133 Wright ME & 2004 Am J Epidemiol & USA & 1 & 1 qui 5 vs 1 & 0.84 \\
\hline 5134 Tsai CJ & 2004 Am J Epidemiol & USA & 1 & 1 qui 5 vs 1 & 1.00 \\
\hline $\begin{array}{l}5135 \text { Zeleniuch- } \\
\text { Jacquotte A }\end{array}$ & 2004 Br J Cancer & USA & 0 & 1 qui 5 vs 1 & 1.70 \\
\hline 5138 Leitzmann MF & 2004 Am J Clin Nutr & USA & 1 & 1 qui 5 vs 1 & 2.02 \\
\hline 5141 Hirvonen T & 2004 Eur J Epidemiol & Finland & 1 & 1 qui 5 vs 1 & 0.86 \\
\hline 5143 Hannan LM & $\begin{array}{c}2004 \text { Cancer Epidemiol } \\
\text { Biomarkers Prev }\end{array}$ & USA & 1 & 1 qui 5 vs 1 & 0.70 \\
\hline 5144 Stack AG & 2004 Kidney Int & USA & 1 & 0 qui 5 vs 1 & 1.44 \\
\hline 5145 Greisenegger S & 2004 Stroke & Austria & 1 & 0 qui 5 vs 1 & 2.60 \\
\hline 5149 Kang JH & 2004 Am J Clin Nutr & USA & 1 & 1 qui 5 vs 1 & 0.90 \\
\hline
\end{tabular}

1 sagittal abdominal diameter
1 alcohol consumption
1 neighborhood-income

1 cis unsaturated fats

1 phylloquinone intake

1 socioeconomic status

1 Income

1 plasma levels of cholesteryl ester transfer protein

1 vegetable consumption

1 retinal arteriolar narrowing

0 Folate intake

0 dietary glycemic load

1 glycemic index

1 current DBP

0 glycemic index

1 milk intake

1 total dietary fiber intake

1 Western pattern

1 Antioxidant diet index

0 dietary total protein intake

0 enterolactone

1 ALA from nonanimal sources

1 Dietary intake of flavonols and

flavones

0 total physical activity

$1 \mathrm{BMI}$

1 mean platelet volume

0 total fat sudden death

atrial fibrillation or

small-for-gestational-

gallstone disease

Coronary heart

disease

hospital mortality

Colorectal

investigation

coronary artery

disease

undiagnosed

hypertension

severe hypertension

Parkinson's disease

cataract extraction

diabetes

Generalized arteriolar narrowing

colorectal adenoma

colorectal cancer

choleystectomy

total strokes

Lung cancer

cholecystectomy

breast cancer

prostate cancer

intermittent

claudication

ovarian cancer

death

severe stroke

primary open-angle glaucoma 
5151 Richardson DB

5153 Holmes MD

5154 Yang $\mathrm{G}$

5155 Klein R

5157 Chen $\mathrm{H}$

5158 Hu FB

5160 Wong TY

5162 Al-Delaimy WK

5163 Jiang $R$

5165 Zylberstein DE

5166 Pell JP

5167 Higginbotham S

5169 McKeown NM

5171 Higginbotham S

5172 Mannisto S

5174 Munger KL

5177 ZeleniuchJacquotte $A$

5178 Mattisson I

5180 Song $Y$

5186 Jiang $R$

$5188 \mathrm{He} \mathrm{K}$

5191 Stattin P

5193 Miller $A B$

5194 Lukanova A

3003 Sornay-Rendu

3004 Pell JP

3006 De Sutter J
2004 Arch Pediatr Adolesc Med USA

2004 Am J Epidemio

2004 Eur J Clin Nutr

USA

USA

2004 Am J Ophthalmol

2004 Am J Epidemio

2004 Diabetes

2004 Ann Intern Med

2004 J Am Coll Nutr

2004 JAMA

2004 Circulation

2004 Am J Epidemiol

2004 J Natl Cancer Inst

2004 Diabetes Care

2004 Cancer Epidemio

Biomarkers Prev

2004 Cancer Epidemiol

2004 Neurology

2004 Br J Cancer

2004 Br J Cancer

2004 Diabetes Care

2004 Am J Clin Nutr

2004 Stroke

2004 Int J Cancer

2004 Int J Cancer

2004 Int J Cancer

2005 J Bone Miner Res

2005 Eur Heart J

2005 Eur Heart J

$\begin{array}{ll}1 \text { qui } 5 \text { vs } 1 & 2.15 \\ 1 \text { qui } 5 \text { vs } 1 & 0.97 \\ 1 \text { qui } 5 \text { vs } 1 & 0.51 \\ 0 \text { qui } 5 \text { vs } 1 & 1.93 \\ 1 \text { qui } 5 \text { vs } 1 & 1.90 \\ 0 \text { qui } 5 \text { vs } 1 & 1.64 \\ 0 \text { qui } 5 \text { vs } 1 & 1.62 \\ 1 \text { qui } 5 \text { vs } 1 & 0.73 \\ 1 \text { qui } 5 \text { vs } 1 & 2.68 \\ 0 \text { qui } 5 \text { vs } & 1.86 \\ 1+2+3+4 & 1.29 \\ 0 \text { qui } 5 \text { vs } & \\ 1+2+3+4 & \\ 1 \text { qui } 5 \text { vs } 1 & 2.85 \\ 0 \text { qui } 5 \text { vs } 1 & 0.62 \\ 1 \text { qui } 5 \text { vs } 1 & 1.01 \\ 1 \text { qui } 5 \text { vs } 1 & 0.98 \\ 1 \text { qui } 5 \text { vs } 1 & 0.67 \\ 1 \text { qui } 5 \text { vs } 1 & 2.49 \\ 0 \text { qui } 5 \text { vs } 1 & 0.58 \\ 0 \text { qui } 5 \text { vs } 1 & 0.89 \\ 1 \text { qui } 5 \text { vs } 1 & 1.28 \\ 0 \text { qui } 5 \text { vs } 1 & 0.71 \\ 1 \text { qui } 5 \text { vs } 1 & 0.80 \\ 0 \text { qui } 5 \text { vs } 1 & 0.60 \\ 1 \text { qui } 5 \text { vs } 1 & 4.76 \\ 0 \text { ter } 3 \text { vs } 1+2 & 1.45 \\ 0 \text { ter } 3 \text { vs } 1 & 0.60\end{array}$

0 ter 3 vs 1
1 adult hemoglobin leve

0 carbohydrate intake

1 intake of tofu and other soy products

1 generalized retinal arteriolar narrowing

1 waist circumference

1 TNF-alphaR2

1 arteriole-to-venule ratios

1 total magnesium intake

1 Ferritin

1 Total Homocysteine

1 Birth weight

1 dietary glycemic load

1 cereal fiber

0 glycemic load

0 beta-Carotene intake

0 total vitamin D intake

1 oestradiol

1 fibre intakes

1 Magnesium intake

1 heme iron from red meat

1 intake of folate

0 total testosterone

1 fruit consumption

1 C-peptide

1 bone loss

1 year of arrest

1 NT-pro-BNP sudden infant death syndrome breast cancer

glycosuria

retinal pigment

epithedepigmentation Parkinson's disease

type 2 diabetes

hypertension

Coronary heart disease

Type 2 diabetes

acut myocardial

infarction

Maternal

events

colorectal cancer

metabolic syndrome

breast cancer risk

lung cancer

multiple sclerosis

breast cancer

breast cancer

Type 2 diabetes

type 2 diabetes

ischemic stroke

prostate cance

lung cancer

Endometrial cancer

fractures

death after

cardiopulmonary

arrest

coronary events 


\begin{tabular}{|c|c|c|c|c|c|c|c|}
\hline 3007 Golden SH & 2005 Psychoneuroendocrinology & USA & 1 & 1 ter 3 vs 1 & 1.34 & $\begin{array}{l}1 \text { trait anger temperament } \\
\text { scores }\end{array}$ & T2DM \\
\hline 3008 Eisner MD & 2005 Thorax & USA & 1 & 0 ter 3 vs 1 & 3.61 & 1 Nicotine exposure & $\begin{array}{l}\text { hospital admission } \\
\text { due to asthma }\end{array}$ \\
\hline 3013 Harville EW & $\begin{array}{l}2005 \text { J Womens Health } \\
\text { (Larchmt) }\end{array}$ & USA & 0 & 1 ter 3 vs 1 & 1.40 & 0 overall stress & Bacterial vaginosis \\
\hline 3014 Khan MM. & 2005 Int J Environ Health Res & Japan & 1 & 0 ter 3 vs 1 & 2.12 & $1 \mathrm{GOT}$ & $\begin{array}{l}\text { longer duration of } \\
\text { having arsenicosis }\end{array}$ \\
\hline 3015 Touvier M & 2005 J Natl Cancer Inst & France & 1 & 1 ter 3 vs 1 & 0.80 & 0 dietary intake of beta carotene & $\begin{array}{l}\text { all-smoking related } \\
\text { cancers }\end{array}$ \\
\hline 3017 Yende S & 2005 Am J Respir Crit Care Med & USA & 1 & 0 ter 3 vs 1 & 1.60 & $1 \mathrm{TNF}$ & $\begin{array}{l}\text { Hospitalization due to } \\
\text { pneumonia }\end{array}$ \\
\hline 3018 Koren-Morag N & 2005 Am J Med & Israel & 1 & 0 ter 3 vs 1 & 1.55 & $1 \mathrm{WBC}$ & $\begin{array}{l}\text { ischemic } \\
\text { cerebrovascular } \\
\text { disease }\end{array}$ \\
\hline 3019 Durairaj L & 2005 Chest & USA & 1 & 1 ter 3 vs 1 & 0.68 & 1 hospital volume & $\begin{array}{l}\text { mortality in GI } \\
\text { diagnoses }\end{array}$ \\
\hline 3020 Kawamoto $\mathrm{R}$ & 2005 Intern Med & Japan & 1 & 0 ter 3 vs 1 & 1.66 & 1 levels of serum uric acid & $\begin{array}{l}\text { intima-media } \\
\text { thickness }\end{array}$ \\
\hline 3023 Tarnow L & $\begin{array}{l}2005 \text { Scand J Clin Lab Invest } \\
\text { Suppl }\end{array}$ & Denmark & 1 & 1 ter 3 vs 1 & 2.64 & 1 PIGF & $\begin{array}{l}\text { cardiovascular } \\
\text { morbidity }\end{array}$ \\
\hline 3024 Schuiling WJ. & 2005 Neurosurgery & Netherlands & 1 & 0 ter 3 vs 1 & 30.9 & $\begin{array}{l}1 \text { acute physiology score II in } \\
\text { subaracnoid hemmorhage } \\
\text { patients }\end{array}$ & $\begin{array}{l}\text { poor outcome after } \\
\text { subarachnoid } \\
\text { hemorrhage }\end{array}$ \\
\hline $\begin{array}{l}3025 \text { Pena de la Vega } \\
\text { L }\end{array}$ & 2005 Mayo Clin Proc & USA & 1 & 0 ter 3 vs $1+2$ & 2.47 & 1 PF4-heparin antibody & all cause mortality \\
\hline $\begin{array}{l}3032 \text { Van Den Brink } \\
\text { CL }\end{array}$ & 2005 Disabil Rehabil & Netherlands & 1 & 1 ter 3 vs 1 & 0.46 & 1 total physical activity & disability \\
\hline 3033 Storti KL & 2005 Prev Med & USA & 1 & 0 ter 3 vs 1 & 1.59 & 1 physical activity & $\begin{array}{l}\text { development of } \\
\text { gallstone disease }\end{array}$ \\
\hline 3034 Wark PA & $\begin{array}{l}2005 \text { Cancer Epidemiol } \\
\text { Biomarkers Prev }\end{array}$ & Netherlands & 1 & 1 ter 3 vs 1 & 0.46 & 1 Fruit consumption & $\begin{array}{l}\text { hMLH1 protein- } \\
\text { deficient colon cancer }\end{array}$ \\
\hline 3036 van der A DL & 2005 Stroke & Netherlands & 1 & 1 ter 3 vs 1 & 1.45 & 0 ferritin levels & stroke \\
\hline 3038 Rashidkhani B & 2005 J Nutr & Sweden & 1 & 1 ter 3 vs 1 & 0.81 & 0 healthy pattern diet & renal cell carcinoma \\
\hline 3039 Boyapati SM & 2005 Breast Cancer Res Treat & USA & 0 & 1 ter 3 vs 1 & 0.99 & 0 soy intake & $\begin{array}{l}\text { disease-free breast } \\
\text { cancer survival }\end{array}$ \\
\hline 3041 Fujiwara Y & $\begin{array}{l}2005 \text { J Gerontol A Biol Sci Med } \\
\text { Sci }\end{array}$ & Japan & 1 & 0 ter 3 vs $1+2$ & 4.70 & 1 Pulse pressure & $\begin{array}{l}\text { Poor cognitive } \\
\text { function }\end{array}$ \\
\hline 3042 Giles LC & $\begin{array}{l}2005 \text { J Epidemiol Community } \\
\text { Health }\end{array}$ & Australia & 1 & 0 ter 3 vs 1 & 0.78 & 1 friends network & survival time \\
\hline 3045 Chiu CJ & 2005 Am J Clin Nutr & USA & 1 & 0 ter 3 vs 1 & 2.46 & 1 carbohydrate intake & cortical opacities \\
\hline 3047 Tripepi G & 2005 J Am Soc Nephrol & Italy & 1 & 0 ter 3 vs 1 & 2.5 & 1 serum IL-6 & all cause mortality \\
\hline 3048 van Hecke MV & 2005 Diabetologia & Netherlands & 1 & 0 ter 3 vs 1 & 2.2 & 1 inflammatory dysfunction & retinopathy \\
\hline
\end{tabular}




\begin{tabular}{|c|c|c|c|c|c|}
\hline 3049 Mangtani P & 2005 Prev Med & UK & 1 & 0 ter 3 vs 1 & 0.87 \\
\hline 3054 Djousse L & 2005 J Am Coll Cardiol & USA & 1 & 1 ter 3 vs 1 & 0.59 \\
\hline 3055 Tseng M & 2005 Am J Clin Nutr & USA & 1 & 1 ter 3 vs 1 & 2.20 \\
\hline 3056 Haumer M & 2005 J Vasc Surg & Austria & 1 & 0 ter 3 vs 1 & 1.83 \\
\hline 3060 Singh JA & 2005 Semin Arthritis Rheum & USA & 1 & 0 ter 3 vs 1 & 1.49 \\
\hline 3065 Salvarani C & 2005 Arthritis Rheum & Italy & 1 & 1 ter 3 vs 1 & 0.11 \\
\hline 3066 Mallamaci F & 2005 Am J Kidney Dis & Italy & 1 & 0 ter 3 vs 1 & 1.72 \\
\hline 3067 Yamagata H & 2005 Diabetes Care & Japan & 1 & 0 ter 3 vs 1 & 3.10 \\
\hline 3070 Wakai K & 2005 Nutr Cancer & Japan & 1 & 1 ter 3 vs 1 & 0.34 \\
\hline 3071 Cherubini A & 2005 Neurobiol Aging & Italy & 1 & 0 ter 3 vs 1 & 2.60 \\
\hline 3072 Weinstein SJ & 2005 J Natl Cancer Inst & USA & 0 & 1 ter 3 vs 1 & 0.49 \\
\hline 3073 Rossi R & 2005 Diabetes Care & Italy & 1 & 0 ter 3 vs 1 & 5.87 \\
\hline 3074 Olijhoek JK & 2005 Diabetes Care & Netherlands & 0 & 1 ter 3 vs 1 & 0.70 \\
\hline 3075 De Bacquer D & 2005 Am J Epidemiol & Belgium & 1 & 0 ter 3 vs 1 & 2.40 \\
\hline 3077 Houston DK & 2005 Am J Clin Nutr & USA & 1 & 0 ter 3 vs 1 & 0.60 \\
\hline $\begin{array}{c}3078 \text { Kurzius-Spencer } \\
\mathrm{M}\end{array}$ & 2005 Pediatr Allergy Immunol & USA & 1 & 0 ter 3 vs 1 & 2.28 \\
\hline 3082 Platek M & $\begin{array}{c}2005 \text { Cancer Epidemiol } \\
\text { Biomarkers Prev }\end{array}$ & USA & 0 & 1 ter 3 vs 1 & 1.60 \\
\hline 3086 Chao A & 2005 JAMA & USA & 1 & 0 ter 3 vs 1 & 1.50 \\
\hline 3087 St-Pierre AC & $\begin{array}{l}2005 \text { Arterioscler Thromb Vasc } \\
\text { Biol }\end{array}$ & Canada & 0 & 1 ter 3 vs 1 & 0.76 \\
\hline 3088 Ulvik A & $\begin{array}{l}2004 \text { Cancer Epidemiol } \\
\text { Biomarkers Prev }\end{array}$ & Norway & 1 & 0 ter 3 vs 1 & 1.32 \\
\hline 3089 Tolmunen T & 2004 Am J Clin Nutr & Finland & 1 & 0 ter 3 vs 1 & 2.30 \\
\hline 3090 Wang AY & 2004 J Am Soc Nephrol & Hong Kong & 1 & 0 ter 3 vs 1 & 4.19 \\
\hline 3093 Schillinger & 2004 Thromb Haemost & Austria & 1 & 0 ter 3 vs $1+2$ & 0.39 \\
\hline 3094 Zeleniuch- & 2005 Int J Cancer & USA & 0 & 1 ter 3 vs 1 & 1.10 \\
\hline
\end{tabular}

\begin{tabular}{|c|c|}
\hline 1 deprivation & $\begin{array}{l}\text { influenza vaccine } \\
\text { uptake }\end{array}$ \\
\hline 1 linolenic acid & $\begin{array}{l}\text { rate-adjustd QT } \\
\text { interval+097 }\end{array}$ \\
\hline 1 dairy food intake & prostate cancer \\
\hline 1 neutrophil counts & $\begin{array}{l}\text { all major } \\
\text { cardiovascular events }\end{array}$ \\
\hline $\begin{array}{l}1 \text { physical component summary } \\
\text { scale }\end{array}$ & any hospitalization \\
\hline 1 levels of ESR & $\begin{array}{l}\text { development of } \\
\text { permanent visual loss }\end{array}$ \\
\hline 1 homocysteine levels & VA thrombosis \\
\hline 1 fasting plama glucose levels & gastric cancer \\
\hline $\begin{array}{l}1 \text { serum carotenoids. retinol. and } \\
\text { tocopherols }\end{array}$ & colorectal cancer \\
\hline 1 plasma vitamin E levels & dementia \\
\hline 0 alpha-tocopherol & prostate cancer \\
\hline $\begin{array}{l}1 \text { flow-mediated dialation of the } \\
\text { branchial artery }\end{array}$ & incident T2DM \\
\hline $\begin{array}{l}0 \text { homeostasis model } \\
\text { assessment of insulin } \\
\text { resistance }\end{array}$ & coronary collaterals \\
\hline 1 social support scale & $\begin{array}{l}\text { coronary heart } \\
\text { disease incidence }\end{array}$ \\
\hline 1 dairy intakes & $\begin{array}{l}\text { impaired activities of } \\
\text { daily living }\end{array}$ \\
\hline 1 serum IgE production & infant eczema \\
\hline 0 serum fructosamine & breast cancer \\
\hline $\begin{array}{l}1 \text { consumption of processed } \\
\text { meat }\end{array}$ & distal colon cancer \\
\hline 0 LDL-C & $\begin{array}{l}\text { ischemic heart } \\
\text { disease }\end{array}$ \\
\hline 1 serum total homocysteine & colorectal cancer \\
\hline $\begin{array}{l}1 \text { serum concentrations of total } \\
\text { homocysteine }\end{array}$ & depression \\
\hline 1 resting energy expenditure & all-cause mortality \\
\hline 1 Heparin cofactor II activity & Instent restenosis \\
\hline 0 levels of estradiol & $\begin{array}{l}\text { breast carcinoma in } \\
\text { situ }\end{array}$ \\
\hline
\end{tabular}

1 deprivation

20

49

11

72

10

60

49

5.87

.70

.40

0.60

28

60

50

76

1.32

1.10
0 levels of estradiol situ 


\begin{tabular}{|c|c|c|c|c|c|}
\hline 3096 Louis GM & 2005 Hum Reprod & USA & 1 & 0 ter 3 vs 1 & 3.77 \\
\hline 3102 Qiu C & 2004 Epidemiology & Sweden & 1 & 1 ter 3 vs 1 & 2.40 \\
\hline 3106 Ramos MI & 2004 Am J Clin Nutr & USA & 1 & 0 ter 3 vs 1 & 2.04 \\
\hline 3110 Qiu C & 2004 Paediatr Perinat Epidemiol & USA & 1 & 0 ter 3 vs 1 & 3.50 \\
\hline 3111 Jowett SL & 2004 Gut & UK & 1 & 0 ter 3 vs 1 & 3.20 \\
\hline 3112 Micheli A & 2004 Int J Cancer & Italy & 1 & 1 ter 3 vs 1 & 2.85 \\
\hline 3113 Rajala U & 2005 J Hum Hypertens & Finland & 1 & 0 ter 3 vs $1+2$ & 3.10 \\
\hline 3115 Ninomiya $T$ & 2004 Am J Kidney Dis & Japan & 1 & 1 ter 3 vs 1 & 2.09 \\
\hline 3116 Nagel G & 2005 Eur J Clin Nutr & Germany & 1 & 0 ter 3 vs 1 & 1.73 \\
\hline 3120 Voutilainen S & 2004 Am J Clin Nutr & Finland & 1 & 1 ter 3 vs 1 & 0.35 \\
\hline 3121 Cohen S & 2004 Psychosom Med & USA & 1 & 0 ter 3 vs 1 & 3.70 \\
\hline 3122 Borugian MJ & $\begin{array}{c}2004 \text { Cancer Epidemiol } \\
\text { Biomarkers Prev }\end{array}$ & Canada & 1 & 1 ter 3 vs 1 & 1.90 \\
\hline $\begin{array}{l}3123 \text { Goderie-Plomp } \\
\text { HW }\end{array}$ & 2004 J Clin Endocrinol Metab & Netherlands & 1 & 0 ter 3 vs 1 & 2.10 \\
\hline 3125 Jansen MC & 2004 Nutr Cancer & Netherlands & 1 & 0 ter 3 vs $1+2$ & 0.64 \\
\hline 3128 Olivot JM & 2004 Stroke & France & 1 & 0 ter 3 vs 1 & 0.56 \\
\hline 3131 Nagata C & 2004 Stroke & Japan & 1 & 1 ter 3 vs 1 & 2.33 \\
\hline 3134 Kiechl S & 2004 Circulation & Austria & 1 & 0 ter 3 vs 1 & 2.20 \\
\hline 3135 Cicuttini FM & 2004 Ann Rheum Dis & Australia & 1 & 0 ter 3 vs 1 & 7.10 \\
\hline 3140 Sieri S & $\begin{array}{l}2004 \text { Cancer Epidemiol } \\
\text { Biomarkers Prev }\end{array}$ & Italy & 1 & 0 ter 3 vs 1 & 0.66 \\
\hline 3142 Zhang $X$ & $\begin{array}{l}2004 \text { Int J Obes Relat Metab } \\
\text { Disord }\end{array}$ & USA & 1 & 1 ter 3 vs 1 & 9.00 \\
\hline 3143 Hadjadj S & 2004 Diabetes Metab & France & 1 & 1 ter 3 vs $1+2$ & 2.01 \\
\hline $\begin{array}{l}3144 \text { Stolzenberg- } \\
\text { Solomon RZ }\end{array}$ & $\begin{array}{l}2004 \text { Cancer Epidemiol } \\
\text { Biomarkers Prev }\end{array}$ & USA & 0 & 1 ter 3 vs 1 & 0.67 \\
\hline 3145 Shankar A & 2004 Am J Hypertens & USA & 1 & 0 ter 3 vs 1 & 1.70 \\
\hline 3147 Mishra GD & 2004 Soc Sci Med & UK & 1 & 0 ter 3 vs 1 & 0.79 \\
\hline
\end{tabular}

1 anti-estrogenic polychlorinated endometriosis biphenyl

0 Extremely-low-frequency magnetic fied lifetime average exposure.

1 folate status

Alzheimer's disease

Center for

Epidemiologic Studies

Depression Scale

(CES-D) score $>l=16$

1 CRP

1 Consumption of meat

gestational diabetes

relapse of ulcerative

colitis

1 free testosterone levels

breast cancer

1 systolic BP

0 total Homocysteine levels

systolic BP

chronic kidney

disease

1 margarine intake

asthma

1 serum folate concentrations

acute coronary events

1 childhood years during which susceptibility to colds their parents owned their home

0 levels of insulin

survival after breast cancer diagnosis

1 serum estradio vertebral fractures

1 Variety in vegetable intake

1 Soluble TM concentration

1 sodium intake

1 level of osteoprotegerin

1 tibial cartilage loss

1 salad vegetables pattern

1 Body mass index

1 triglycerlde levels

0 IGF-1

1 WBC count

1 socioeconomic status
Total cancers

brain infarctions

death from total stroke

cardiovascular

disease

knee replacement

breast cancer

$\mathrm{CHD}$

rogression of diabetic

nephropathy

pancreatic cance

Hypertension

death 


\begin{tabular}{|c|c|c|c|c|c|c|c|}
\hline 3148 Johansson L & 2004 Stroke & Sweden & 1 & 0 ter 3 vs 1 & 0.27 & 1 level of VWF & $\begin{array}{l}\text { intracerebral } \\
\text { hemorrhage }\end{array}$ \\
\hline 3149 Ala-Mursula L & 2004 Occup Environ Med & Finland & 1 & 0 ter 3 vs 1 & 1.90 & 1 worktime control & poor self rated health \\
\hline 3150 Haim M & 2004 Arch Intern Med & Israel & 1 & 0 ter 3 vs 1 & 1.47 & 1 WBC count & total mortality \\
\hline 3153 Sanchez PL & 2004 Heart & Spain & 1 & 0 ter 3 vs 1 & 4.51 & $1 \mathrm{C}$ reactive protein & cardiovascular death \\
\hline 3154 Ballantyne CM & 2004 Circulation & USA & 1 & 0 ter 3 vs 1 & 1.78 & 1 Lp-PLA2 & incident CHD \\
\hline 3157 Tseng M & $\begin{array}{c}2004 \text { Cancer Epidemiol } \\
\text { Biomarkers Prev }\end{array}$ & USA & 0 & 1 ter 3 vs 1 & 0.60 & 0 Southern pattern diet & prostate cancer \\
\hline 3160 Merlino LA & 2004 Arthritis Rheum & USA & 1 & 1 ter 3 vs 1 & 0.67 & 1 vitamin $D$ intake & rheumatoid arthritis \\
\hline 3162 Meyerhardt JA & 2004! J Clin Oncol & USA & 1 & 1 ter 3 vs 1 & 1.94 & 1 hospital surgical volume & cancer recurrence \\
\hline 2002 Bak H & 2005 Int J Cancer & Denmark & 1 & 1 medians & 1.61 & 1 DNA adduct levels & lung cancer \\
\hline $\begin{array}{l}2011 \text { Polychronopoulos } \\
\text { E }\end{array}$ & 2005 Lipids Health Dis & Greece & 1 & 0 medians & 0.77 & 1 Mediterranean diet & hypercholesterolemia \\
\hline 2021 Olson MB & 2005 Psychosom Med & USA & 1 & 0 medians & 1.35 & $\begin{array}{l}1 \text { total Cook Medley Hostility } \\
\text { Score }\end{array}$ & adverse event \\
\hline 2038 Verma A & 2005 Heart & USA & 1 & 0 medians & 2.19 & 1 single pre-implant BNP level & ICD therapies \\
\hline 2048 Shalak LF & 2005 J Perinatol & USA & 1 & 0 medians & 2.80 & 1 rectal temperature & admission to NICU \\
\hline 2051 Ceschi M & 2005 Carcinogenesis & Switzerland & 1 & 0 medians & 0.67 & 1 intake level of n- 6 fatty acids & breast cancer \\
\hline 2065 George DJ & 2005 Clin Cancer Res & USA & 1 & 0 medians & 1.38 & 1 Plasma IL-6 level & $\begin{array}{l}\text { hormone-refractory } \\
\text { prostate cancer } \\
\text { progression and } \\
\text { patient survival }\end{array}$ \\
\hline 2088 Gaede P & 2005 Diabetologia & Denmark & 1 & 0 medians & 4.40 & $\begin{array}{l}1 \text { plasma } \mathrm{N} \text {-terminal probrain } \\
\text { natriuretic peptide }\end{array}$ & $\begin{array}{l}\text { cardiovascular } \\
\text { disease }\end{array}$ \\
\hline 2089 Tarnow L & 2005 Diabetologia & Denmark & 1 & 0 medians & 3.86 & $\begin{array}{l}1 \text { plasma } \mathrm{N} \text {-terminal probrain } \\
\text { natriuretic peptide }\end{array}$ & any cause death \\
\hline 2112 Tolmunen T & 2004 Psychother Psychosom & Finland & 1 & 0 medians & 3.04 & 1 intake of folate & $\begin{array}{l}\text { depression } \\
\text { diagnosis }\end{array}$ \\
\hline 2172 Mayne ST & 2004 J Am Coll Nutr & USA & 1 & 0 medians & 0.53 & 1 plasma lycopene & total mortality \\
\hline
\end{tabular}




\section{References:}

1 Adebamowo CA CE. Sampson L. Katan MB. Spiegelman D. Willett WC. Holmes MD. Dietary flavonols and flavonol-rich foods intake and the risk of breast cancer. Int J Cancer. 2005;114:628-633.

2 Al Snih S MK. Ottenbacher KJ. Raji MA. Hand grip strength and incident ADL disability in elderly Mexican Americans over a seven-year period. Aging Clin Exp Res. 2004;16:481-486.

3 Ala-Mursula L VJ. Pentti J. Kivimaki M. Effect of employee worktime control on health: a prospective cohort study. Occup Environ Med. 2004;61:254-261.

4 Al-Delaimy WK RE. Willett WC. Stampfer MJ. Hu FB. Magnesium intake and risk of coronary heart disease among men. J Am Coll Nutr. 2004;23:6370.

5 Alonso A dlFC. Martin-Arnau AM. de Irala J. Martinez JA. MartinezGonzalez MA. Fruit and vegetable consumption is inversely associated with blood pressure in a Mediterranean population with a high vegetable-fat intake: the Seguimiento Universidad de Navarra (SUN) Study. Br J Nutr. 2004;92:311-319.

6 Alonso A M-GM. Olive oil consumption and reduced incidence of hypertension: the SUN study. Lipids. 2005;39:1233-1238.

7 Anderson JP RJ. Folsom AR. Anthropometric variables. physical activity. and incidence of ovarian cancer: The Iowa Women's Health Study. Cancer. 2004;100:1515-1521.

8 Arenillas JF MC. Chacon P. Rovira A. Montaner J. Coscojuela P. Sanchez E. Quintana M. Alvarez-Sabin J. High lipoprotein (a). diabetes. and the extent of symptomatic intracranial atherosclerosis. Neurology. 2004;63:27-32.

9 Bak H AH. Thomsen BL. Tjonneland A. Overvad K. Vogel U. RaaschouNielsen O. Loft S. Bulky DNA adducts as risk indicator of lung cancer in a Danish case-cohort study. Int J Cancer. 2006;118:1618-1622.

10 Ballantyne CM HR. Bang H. Coresh J. Folsom AR. Heiss G. Sharrett AR. Lipoprotein-associated phospholipase A2. high-sensitivity C-reactive protein. and risk for incident coronary heart disease in middle-aged men and women in the Atherosclerosis Risk in Communities (ARIC) study. Circulation. 2004;109:837-842.

11 Batty GD ME. Osler M. Childhood IQ in relation to later psychiatric disorder: evidence from a Danish birth cohort study. Br J Psychiatry. 2005;187:180181.

12 Baumgartner KB HW. Baumgartner RN. Crumley DD. Gilliland FD. McTiernan A. Bernstein L. Ballard-Barbash R. Association of body composition and weight history with breast cancer prognostic markers: divergent pattern for Hispanic and non-Hispanic White women. Am J Epidemiol. 2004;160:1087-1097.

13 Bautista LE VL. Arenas IA. Gamarra G. Independent association between inflammatory markers (C-reactive protein. interleukin-6. and TNF-alpha) and essential hypertension. J Hum Hypertens. 2005;19:149-154.

14 Beddhu S RN. Pappas LM. Normalization of protein intake by body weight and the associations of protein intake with nutritional status and survival. $J$ Ren Nutr. 2005;15:387-397. 
15 Benner JS PM. Smith TW. Bullano MF. Willey VJ. Williams SA. Association between short-term effectiveness of statins and long-term adherence to lipidlowering therapy. Am J Health Syst Pharm. 2005;62:1468-1475.

16 Bergelt C CJ. Prescott E. Gronbaek M. Koch U. Johansen C. Vital exhaustion and risk for cancer: a prospective cohort study on the association between depressive feelings. fatigue. and risk of cancer. Cancer Causes Control. 2005;104:1288-1295.

17 Bergink AP UA. Van Leeuwen JP. Hofman A. Verhaar JA. Pols HA. Bone mineral density and vertebral fracture history are associated with incident and progressive radiographic knee osteoarthritis in elderly men and women: The Rotterdam Study. Bone. 2005;37:446-456.

18 Berndt SI CH. Landis PK. Hallfrisch J. Rohrmann S. Metter EJ. Platz EA. Prediagnostic plasma vitamin $C$ levels and the subsequent risk of prostate cancer. Nutrition. 2005;21:686-690.

19 Bertone-Johnson ER CW. Holick MF. Hollis BW. Colditz GA. Willett WC. Hankinson SE. Plasma 25-hydroxyvitamin D and 1.25-dihydroxyvitamin D and risk of breast cancer. Cancer Epidemiol Biomarkers Prev. 2005;14:19911997.

20 Bertone-Johnson ER HS. Bendich A. Johnson SR. Willett WC. Manson JE. Calcium and vitamin D intake and risk of incident premenstrual syndrome. Arch Intern Med. 2005;165(11):1246-1252.

21 Blake GJ PA. Manson JE. Williams GR. Buring J. Ridker PM. Glynn RJ. Hemoglobin A1c level and future cardiovascular events among women. Arch Intern Med. 2004;164:757-761.

22 Boekholdt SM KJ. Wareham NJ. Peters RJ. Jukema JW. Luben R. Bingham SA. Day NE. Kastelein JJ. Khaw KT. Plasma levels of cholesteryl ester transfer protein and the risk of future coronary artery disease in apparently healthy men and women: the prospective EPIC (European Prospective Investigation into Cancer and nutrition)-Norfolk population study. Circulation. 2004;110:1418-1423.

23 Boekholdt SM PR. Day NE. Luben R. Bingham SA. Wareham NJ. Hack CE. Reitsma PH. Khaw KT. Macrophage migration inhibitory factor and the risk of myocardial infarction or death due to coronary artery disease in adults without prior myocardial infarction or stroke: the EPIC-Norfolk Prospective Population study. Am J Med. 2004;117:390-397.

24 Boekholdt SM PR. Hack CE. Day NE. Luben R. Bingham SA. Wareham NJ. Reitsma PH. Khaw KT. IL-8 plasma concentrations and the risk of future coronary artery disease in apparently healthy men and women: the EPICNorfolk prospective population study. Arterioscler Thromb Vasc Biol. 2004;24:1503-1508.

25 Borugian MJ SS. Kim-Sing C. Van Patten C. Potter JD. Dunn B. Gallagher RP. Hislop TG. Insulin. macronutrient intake. and physical activity: are potential indicators of insulin resistance associated with mortality from breast cancer? Cancer Epidemiol Biomarkers Prev. 2004;13:1163-1172.

26 Bower M GB. Mandalia S. Newsom-Davis T. Thirlwell C. Dhillon T. Young AM. Powles T. Gaya A. Nelson M. Stebbing J. A prognostic index for systemic AIDS-related non-Hodgkin lymphoma treated in the era of highly active antiretroviral therapy. Ann Intern Med. 2005;143:265-273. 
27 Boyapati SM SX. Ruan ZX. Dai Q. Cai Q. Gao YT. Zheng W. Soyfood intake and breast cancer survival: a followup of the Shanghai Breast Cancer Study. Breast Cancer Res Treat. . 2005;92:11-17.

28 Bruno G CF. Merletti F. Cavallo-Perin P. Gandolfo E. Rivetti M. Runzo C. Pinach S. Pagano G; Piedmont Study Group for Diabetes Epidemiology. Residual beta-cell function and male/female ratio are higher in incident young adults than in children: the registry of type 1 diabetes of the province of Turin. Italy. 1984-2000. Diabetes Care. 2005;28:312-317.

29 Butler LM. Koh WP. Lee HP. Tseng M. Yu MC. London SJ. Prospective Study of Dietary Patterns and Persistent Cough with Phlegm among Chinese Singaporeans. Am J Respir Crit Care Med. Feb 1 2006;173:264-270.

30 Butler LM KW. Lee HP. Yu MC. London SJ. Dietary fiber and reduced cough with phlegm: a cohort study in Singapore. Am J Respir Crit Care Med. 2004;170:279-287.

31 Ceschi M SC. Van Den Berg D. Koh WP. Yu MC. Probst-Hensch N. The effect of cyclin D1 (CCND1) G870A-polymorphism on breast cancer risk is modified by oxidative stress among Chinese women in Singapore. Carcinogenesis. . 2005;26:1457-1464.

32 Chao A TM. Connell CJ. McCullough ML. Jacobs EJ. Flanders WD. Rodriguez C. Sinha R. Calle EE. Meat consumption and risk of colorectal cancer. JAMA. 2005;293:172-182.

33 Chen C LS. Voigt L. Fitzpatrick A. Plymate SR. Weiss NS. Prostate carcinoma incidence in relation to prediagnostic circulating levels of insulinlike growth factor I. insulin-like growth factor binding protein 3. and insulin. Cancer. 2005;103:76-84.

34 Chen H ZS. Schwarzschild MA. Hernan MA. Logroscino G. Willett WC. Ascherio A. Folate intake and risk of Parkinson's disease. Am J Epidemiol. 2004;160:368-375.

35 Chen H ZS. Schwarzschild MA. Hernan MA. Willett WC. Ascherio A. Obesity and the risk of Parkinson's disease. Am J Epidemiol. 2004;159:547555.

36 Cherubini A MA. Andres-Lacueva C. Di Iorio A. Lamponi M. Mecocci P. Bartali B. Corsi A. Senin U. Ferrucci L. Vitamin E levels. cognitive impairment and dementia in older persons: the InCHIANTI study. Neurobiol Aging. 2005;26:987-994.

37 Chiu CJ MM. Rogers G. Jacques PF. Chylack LT Jr. Tung W. Hankinson SE. Willett WC. Taylor A. Carbohydrate intake and glycemic index in relation to the odds of early cortical and nuclear lens opacities. Am J Clin Nutr. 2005;81:1411-1416.

38 Cho E S-WS. Spiegelman D. Beeson WL. van den Brandt PA. Colditz GA. Folsom AR. Fraser GE. Freudenheim JL. Giovannucci E. Goldbohm RA. Graham S. Miller AB. Pietinen P. Potter JD. Rohan TE. Terry P. Toniolo P. Virtanen MJ. Willett WC. Wolk A. Wu K. Yaun SS. Zeleniuch-Jacquotte A. Hunter DJ. Dairy foods. calcium. and colorectal cancer: a pooled analysis of 10 cohort studies. J Natl Cancer Inst. 2004;96:1015-1022.

39 Church TS CY. Earnest CP. Barlow CE. Gibbons LW. Priest EL. Blair SN. Exercise capacity and body composition as predictors of mortality among men with diabetes. Diabetes Care. 2004;27:83-88. 
40 Cicuttini FM JG. Forbes A. Wluka AE. Rate of cartilage loss at two years predicts subsequent total knee arthroplasty: a prospective study. Ann Rheum Dis. . 2004;63:1124-1127.

41 Cocco P FD. Billai B. D'Atri M. Melis M. Blair A. Cancer mortality among men occupationally exposed to dichlorodiphenyltrichloroethane. Cancer Res. 2005;65:9588-9594.

42 Cohen S DW. Turner RB. Alper CM. Skoner DP. Childhood socioeconomic status and host resistance to infectious illness in adulthood. Psychosom Med. . 2004;66:553-558.

43 Dallaire F DE. Muckle G. Vezina C. Jacobson SW. Jacobson JL. Ayotte P. Acute infections and environmental exposure to organochlorines in Inuit infants from Nunavik. Environ Health Perspect. 2004;112:1359-1365.

44 De Bacquer D PE. Clays E. Mak R. Moreau M. de Smet P. Kornitzer M. De Backer G. Perceived job stress and incidence of coronary events: 3-year follow-up of the Belgian Job Stress Project cohort. Am J Epidemiol. 2005;161:434-441.

45 De Cosmo S MA. Ludovico O. Mastroianno S. Di Giorgio A. Pirro L. Trischitta V. Increased urinary albumin excretion. insulin resistance. and related cardiovascular risk factors in patients with type 2 diabetes: evidence of a sex-specific association. Diabetes Care. 2005;28:910-915.

46 De Michele M IA. Panico S. Celentano E. Sacchetti L. Mazzaccara C. Salvato A. Bond MG. Salvatore F. Rubba P. Effect of high-density lipoprotein cholesterol levels on carotid artery geometry in a Mediterranean female population. Eur J Cardiovasc Prev Rehabil. 2004;11:403-407.

47 De Sutter J. De Bacquer D. Cuypers S. et al. Plasma N-terminal pro-brain natriuretic peptide concentration predicts coronary events in men at work: a report from the BELSTRESS study. Eur Heart J. Dec 2005;26(24):2644-2649.

48 Dijk JM vdGY. Grobbee DE. Bots ML; SMART Study Group. Carotid stiffness indicates risk of ischemic stroke and TIA in patients with internal carotid artery stenosis: the SMART study. Stroke. 2004;35:2258-2262.

49 Djousse L RP. Hopkins PN. Whitsel EA. Arnett DK. Eckfeldt JH. Province MA. Ellison RC; Investigators of the NHLBI Family Heart Study. Dietary linolenic acid and adjusted QT and JT intervals in the National Heart. Lung. and Blood Institute Family Heart study. J Am Coll Cardiol. 2005;45:17161722.

50 Duncan BB SM. Pankow JS. Bang H. Couper D. Ballantyne CM. Hoogeveen RC. Heiss G. Adiponectin and the development of type 2 diabetes: the atherosclerosis risk in communities study. Diabetes. 2004;53:2473-2478.

51 Durairaj L TJ. Chrischilles EA. Vaughan Sarrazin MS. Yankey J. Rosenthal GE. Hospital volume-outcome relationships among medical admissions to ICUs. Chest. 2005;128:1682-1689.

52 Eisner MD KJ. Hammond SK. Koren G. Lactao G. Iribarren C. Directly measured second hand smoke exposure and asthma health outcomes. Thorax. 2005;60:814-821.

53 Elkind MS SR. Boden-Albala B. Rundek T. Paik MC. Sacco RL. Relative elevation in baseline leukocyte count predicts first cerebral infarction. Neurology. 2005;64:2121-2125.

54 Elkins JS OME. Longstreth WT Jr. Carlson MC. Manolio TA. Johnston SC. Stroke risk factors and loss of high cognitive function. Neurology. 2004;63:793-799. 
55 Empana JP DP. Charles MA. Jouven X. Sagittal abdominal diameter and risk of sudden death in asymptomatic middle-aged men: the Paris Prospective Study I. Circulation. . 2004;110:2781-2785.

56 English DR MR. Hodge AM. Hopper JL. Haydon AM. Giles GG. Red meat. chicken. and fish consumption and risk of colorectal cancer. Cancer Epidemiol Biomarkers Prev. 2004;13:1509-1514.

57 Engstrom G BG. Lindblad B. Janzon L. Lindgarde F. Incidence of fatal or repaired abdominal aortic aneurysm in relation to inflammation-sensitive plasma proteins. Arterioscler Thromb Vasc Biol. 2004;24:337-341.

58 Engstrom G HB. Eriksson KF. Janzon L. Lindgarde F. Complement C3 is a risk factor for the development of diabetes: a population-based cohort study. Diabetes. 2005;54:570-575.

59 Engstrom G S-FD. Lindblad B. Janzon L. Lindgarde F. Risk of treatment of peripheral arterial disease is related to inflammation-sensitive plasma proteins: a prospective cohort study. J Vasc Surg. 2004;40:1101-1105.

60 Erkkila AT BS. Hu FB. Jacques PF. Manson JE. Rexrode KM. Stampfer MJ. Lichtenstein AH. Phylloquinone intake as a marker for coronary heart disease risk but not stroke in women. Eur J Clin Nutr. 2005;59(2):196-204.

61 Erlinger TP PE. Rifai N. Helzlsouer KJ. C-reactive protein and the risk of incident colorectal cancer. JAMA. 2004;291:589-590.

62 Esmaillzadeh A MP. Azizi F. Whole-grain consumption and the metabolic syndrome: a favorable association in Tehranian adults. Eur J Clin Nutr. 2005;59:353-362.

63 Flood A PU. Chatterjee N. Lacey JV Jr. Schairer C. Schatzkin A. Calcium from diet and supplements is associated with reduced risk of colorectal cancer in a prospective cohort of women. Cancer Epidemiol Biomarkers Prev. 2005;14:126-132.

64 Folsom AR DZ. Fish intake. marine omega-3 fatty acids. and mortality in a cohort of postmenopausal women. Am J Epidemiol. 2004;160:1005-1010.

65 Ford ES MA. Liu S. Healthy Eating Index and C-reactive protein concentration: findings from the National Health and Nutrition Examination Survey III. 1988-1994. Eur J Clin Nutr. 2005;59:278-283.

66 Forman JP B-FH. Willett WC. Stampfer MJ. Curhan GC. Vitamin D intake and risk of incident hypertension: results from three large prospective cohort studies. Hypertension. 2005;46:676-682.

67 Fried LF. Katz R. Sarnak MJ. et al. Kidney function as a predictor of noncardiovascular mortality. J Am Soc Nephrol. 2005;16:3728-3735.

68 Frost L VP. Alcohol and risk of atrial fibrillation or flutter: a cohort study. Arch Intern Med. 2004;164:1993-1998.

69 Frost L VP. Caffeine and risk of atrial fibrillation or flutter: the Danish Diet. Cancer. and Health Study. Am J Clin Nutr. 2005;81:578-582.

70 Frost L VP. n-3 Fatty acids consumed from fish and risk of atrial fibrillation or flutter: the Danish Diet. Cancer. and Health Study. Am J Clin Nutr. 2005;81:50-54.

71 Fujiwara Y CP. Takahashi R. Amano H. Yoshida H. Kumagai S. Fujita K. Wang DG. Shinkai S. Arterial pulse wave velocity as a marker of poor cognitive function in an elderly community-dwelling population. J Gerontol A Biol Sci Med Sci. . 2005;60:607-612. 
72 Fung TT HF. Holmes MD. Rosner BA. Hunter DJ. Colditz GA. Willett WC. Dietary patterns and the risk of postmenopausal breast cancer. Int J Cancer. 2005;116:116-121.

73 Fung TT SM. Manson JE. Willett WC. Hu FB. Dietary patterns. meat intake. and the risk of type 2 diabetes in women. Arch Intern Med. 2004;164:22352240.

74 Fung TT SM. Manson JE. Rexrode KM. Willett WC. Hu FB. Prospective study of major dietary patterns and stroke risk in women. Stroke. 2004;35:2014-2019.

75 Gaede P HP. Hess G. Parving HH. Pedersen O. Plasma N-terminal pro-brain natriuretic peptide as a major risk marker for cardiovascular disease in patients with type 2 diabetes and microalbuminuria. Diabetologia. 2005;48(1):156163.

76 Gago-Dominguez M CJ. Sun CL. Van Den Berg D. Koh WP. Lee HP. Yu MC. Marine n-3 fatty acid intake. glutathione S-transferase polymorphisms and breast cancer risk in post-menopausal Chinese women in Singapore. Carcinogenesis. 2004;25:2143-2147.

77 Garcia-Palmieri MR CC. Mc Gee D. Sempos C. Smit E. Sorlie PD. Wide pulse pressure is an independent predictor of cardiovascular mortality in Puerto Rican men. Nutr Metab Cardiovasc Dis. 2005;15(1):71-78.

78 Manson JE. Buring JE. Levey AS. Gaziano JM. Association between body mass index and CKD in apparently healthy men. Am J Kidney Di. 2005;46:871-80

79 George DJ HS. Shepard TF. Sanford B. Vogelzang NJ. Small EJ. Kantoff PW. The prognostic significance of plasma interleukin-6 levels in patients with metastatic hormone-refractory prostate cancer: results from cancer and leukemia group B 9480. Clin Cancer Res. . 2005;11:1815-1820.

80 Gilbert PB PM. Follmann D. Hudgens MG. Francis DP. Gurwith M. Heyward WL. Jobes DV. Popovic V. Self SG. Sinangil F. Burke D. Berman PW. Correlation between immunologic responses to a recombinant glycoprotein 120 vaccine and incidence of HIV-1 infection in a phase $3 \mathrm{HIV}-1$ preventive vaccine trial. J Infect Dis. 2005;191:666-677.

81 Giles LC GG. Luszcz MA. Andrews GR. Effect of social networks on 10 year survival in very old Australians: the Australian longitudinal study of aging. $J$ Epidemiol Community Health. . 2005;59:574-579.

82 Giltay EJ GJ. Zitman FG. Hoekstra T. Schouten EG. Dispositional optimism and all-cause and cardiovascular mortality in a prospective cohort of elderly dutch men and women. Arch Gen Psychiatry. 2004;61:1126-1135.

83 Goderie-Plomp HW vdKM. de Ronde W. Hofman A. de Jong FH. Pols HA. Endogenous sex hormones. sex hormone-binding globulin. and the risk of incident vertebral fractures in elderly men and women: the Rotterdam Study. $J$ Clin Endocrinol Metab. 2004;89:3261-3269.

84 Golden SH. Williams JE. Ford DE. et al. Anger temperament is modestly associated with the risk of type 2 diabetes mellitus: The atheroslcerosis risk in communities study. Psychoneuroendocrinology. 2005.

85 Golden SH WJ. Ford DE. Yeh HC. Paton Sanford C. Nieto FJ. Brancati FL; Atherosclerosis Risk in Communities study. Depressive symptoms and the risk of type 2 diabetes: the Atherosclerosis Risk in Communities study. Diabetes Care. 2004;27:429-435. 
86 Greisenegger S EG. Hsieh K. Tentschert S. Mannhalter C. Lalouschek W. Is elevated mean platelet volume associated with a worse outcome in patients with acute ischemic cerebrovascular events? Stroke. 2004;35:1688-1691.

87 Guerra S LI. Halonen M. Martinez FD. Wright AL. Reduced interferon gamma production and soluble CD14 levels in early life predict recurrent wheezing by 1 year of age. Am J Respir Crit Care Med. 2004;169:70-76.

88 Gunnarsdottir I BB. Benediktsson R. Gudnason V. Thorsdottir I. Association between size at birth. truncal fat and obesity in adult life and its contribution to blood pressure and coronary heart disease; study in a high birth weight population. Eur J Clin Nutr. 2004;58:812-818.

89 Hadjadj S D-BB. Bekherraz A. BrIdoux F. Gallois Y. Mauco G. Ebran J. Marre M. Serum triglycerides are a predictive factor for the development and the progression of renal and retinal complications in patients with type 1 diabetes. Diabetes Metab. . 2004;30:43-51.

90 Haim M BV. Goldbourt U. Battler A. Behar S. Predictive value of elevated white blood cell count in patients with preexisting coronary heart disease: the Bezafibrate Infarction Prevention Study. Arch Intern Med. 2004;164:433-439.

91 Hannan LM LM. Lacey JV Jr. Colbert LH. Albanes D. Schatzkin A. Schairer C. Physical activity and risk of ovarian cancer: a prospective cohort study in the United States. Cancer Epidemiol Biomarkers Prev. 2004;13:765-770.

92 Hartman TJ AP. Snyder K. Slattery ML. Caan B. Paskett E. Iber F. Kikendall JW. Marshall J. Shike M. Weissfeld J. Brewer B. Schatzkin A. Lanza E; Polyp Prevention Study Group. The association of calcium and vitamin D with risk of colorectal adenomas. J Nutr. 2005;135:252-259.

93 Harville EW HM. Zhang J. Perceived life stress and bacterial vaginosis. $J$ Womens Health (Larchmt). . 2005;14:627-633.

94 Haumer M AJ. Exner M. Mlekusch W. Sabeti S. Schlager O. Schwarzinger I. Wagner O. Minar E. Schillinger M. Association of neutrophils and future cardiovascular events in patients with peripheral artery disease. J Vasc Surg. 2005;41:610-617.

95 Hayashi T BE. Leonetti DL. McNeely MJ. Newell-Morris L. Kahn SE. Fujimoto WY. Visceral adiposity is an independent predictor of incident hypertension in Japanese Americans. Ann Intern Med. 2004;140:992-1000.

96 He K MA. Rimm EB. Rosner BA. Stampfer MJ. Willett WC. Ascherio A. Folate. vitamin B6. and B12 intakes in relation to risk of stroke among men. Stroke. 2004;35:169-174.

97 Hedblad B OM. Engstrom G. Wollmer P. Janzon L. Heterogeneity of cardiovascular risk among smokers is related to degree of carbon monoxide exposure. Atherosclerosis. 2005;179:177-183.

98 Heidemann C HK. Spranger J. Klipstein-Grobusch K. Mohlig M. Pfeiffer AF. Boeing H; European Prospective Investigation into Cancer and Nutrition (EPIC)--Potsdam Study Cohort. A dietary pattern protective against type 2 diabetes in the European Prospective Investigation into Cancer and Nutrition (EPIC)--Potsdam Study cohort. Diabetologia. 2005;48:1126-1134.

99 Higginbotham S ZZ. Lee IM. Cook NR. Giovannucci E. Buring JE. Liu S; Women's Health Study. Dietary glycemic load and risk of colorectal cancer in the Women's Health Study. J Natl Cancer Inst. 2004;96:229-233.

100 Higginbotham S ZZ. Lee IM. Cook NR. Buring JE. Liu S. Dietary glycemic load and breast cancer risk in the Women's Health Study. Cancer Epidemiol Biomarkers Prev. 2004;13:65-70. 
101 Hill DJ HC. Food allergy and atopic dermatitis in infancy: an epidemiologic study. Pediatr Allergy Immunol. 2004;15:421-427.

102 Hirvonen T TM. Pietinen P. Korhonen P. Albanes D. Virtamo J. Flavonol and flavone intake and the risk of intermittent claudication in male smokers. Eur $J$ Epidemiol. 2004;19:305-311.

103 Hoffmeister A RD. Kunze M. Brenner H. Koenig W. Prognostic value of inflammatory markers alone and in combination with blood lipids in patients with stable coronary artery disease. Eur J Intern Med. 2005;16:47-52.

104 Holmes MD LS. Hankinson SE. Colditz GA. Hunter DJ. Willett WC. Dietary carbohydrates. fiber. and breast cancer risk. Am J Epidemiol. 2004;159:732-9.

105 Horne BD MJ. Lappe DL. Renlund DG. Bair TL. Bunch TJ. Anderson JL; Intermountain Heart Collaborative Study Group. Less affluent area of residence and lesser-insured status predict an increased risk of death or myocardial infarction after angiographic diagnosis of coronary disease. Ann Epidemiol. 2004;14:143-150.

106 Houston DK SJ. Cai J. Haines PS. Dairy. fruit. and vegetable intakes and functional limitations and disability in a biracial cohort: the Atherosclerosis Risk in Communities Study. Am J Clin Nutr. . 2005;81:1454.

107 Hu FB MJ. Li TY. Rifai N. Manson JE. Inflammatory markers and risk of developing type 2 diabetes in women. Diabetes. 2004;53:693-700.

108 Hubbard JS RS. Landis PK. Metter EJ. Muller DC. Andres R. Carter HB. Platz EA. Association of prostate cancer risk with insulin. glucose. and anthropometry in the Baltimore longitudinal study of aging. Urology. 2004;63:253-258.

109 Hung HC JK. Jiang R. Hu FB. Hunter D. Smith-Warner SA. Colditz GA. Rosner B. Spiegelman D. Willett WC. Fruit and vegetable intake and risk of major chronic disease. J Natl Cancer Inst. 2004;96:1577-1584.

110 Hutchings A RR. Brady A. Wildman M. Rowan K. Socioeconomic status and outcome from intensive care in England and Wales. Med Care. 2004;42:943951.

111 Ito Y WK. Suzuki K. Ozasa K. Watanabe Y. Seki N. Ando M. Nishino Y. Kondo T. Ohno Y. Tamakoshi A; JACC Study Group. Lung cancer mortality and serum levels of carotenoids. retinol. tocopherols. and folic acid in men and women: a case-control study nested in the JACC Study. J Epidemiol. 2005;15:S140-149.

112 Jansen MC B-d-MH. Feskens EJ. Streppel MT. Kok FJ. Kromhout D. Quantity and variety of fruit and vegetable consumption and cancer risk. Nutr Cancer. . 2004;48:142-148.

113 Jee SH. Park JY. Kim HS. Lee TY. Samet JM. White blood cell count and risk for all-cause. cardiovascular. and cancer mortality in a cohort of Koreans. Am J Epidemiol. Dec 1 2005;162:1062-1069.

114 Jensen MK K-BP. Hu FB. Franz M. Sampson L. Gronbaek M. Rimm EB. Intakes of whole grains. bran. and germ and the risk of coronary heart disease in men. Am J Clin Nutr. 2004;80:1492-1499.

115 Jiang R MJ. Meigs JB. Ma J. Rifai N. Hu FB. Body iron stores in relation to risk of type 2 diabetes in apparently healthy women. JAMA. 2004;291:711717.

116 Jiang R MJ. Ascherio A. Stampfer MJ. Willett WC. Hu FB. Dietary iron intake and blood donations in relation to risk of type 2 diabetes in men: a prospective cohort study. Am J Clin Nutr. 2004;79:70-75. 
117 Jiang R SM. Li T. Rifai N. Stampfer MJ. Rimm EB. Hu FB. Non-HDL cholesterol and apolipoprotein B predict cardiovascular disease events among men with type 2 diabetes. Diabetes Care. 2004;27:1991-1997.

118 Johansson L JJ. Stegmayr B. Nilsson TK. Hallmans G. Boman K. Hemostatic factors as risk markers for intracerebral hemorrhage: a prospective incident case-referent study. Stroke. 2004;35:826-830.

119 Jowett SL SC. Pearce MS. Phillips E. Gregory W. Barton JR. Welfare MR. Influence of dietary factors on the clinical course of ulcerative colitis: a prospective cohort study. GUT. 2004;53:1479-1484.

120 Kaaks R BF. Key T. Rinaldi S. Dossus L. Biessy C. Secreto G. Amiano P. Bingham S. Boeing H. Bueno de Mesquita HB. Chang-Claude J. ClavelChapelon F. Fournier A. van Gils CH. Gonzalez CA. Gurrea AB. Critselis E. Khaw KT. Krogh V. Lahmann PH. Nagel G. Olsen A. Onland-Moret NC. Overvad K. Palli D. Panico S. Peeters P. Quiros JR. Roddam A. Thiebaut A. Tjonneland A. Chirlaque MD. Trichopoulou A. Trichopoulos D. Tumino R. Vineis P. Norat T. Ferrari P. Slimani N. Riboli E. Serum sex steroids in premenopausal women and breast cancer risk within the European Prospective Investigation into Cancer and Nutrition (EPIC). J Natl Cancer Inst. 2005;97:755-765.

121 Kado DM KA. Huang MH. Troen A. Rowe JW. Selhub J. Seeman TE. Homocysteine versus the vitamins folate. B6. and B12 as predictors of cognitive function and decline in older high-functioning adults: MacArthur Studies of Successful Aging. Am J Med. 2005;118:161-167.

122 Kahn RS BD. Whitaker RC. Combined effect of mothers' and fathers' mental health symptoms on children's behavioral and emotional well-being. Arch Pediatr Adolesc Med 2004;1588(8):721-729.

123 Kang JH PL. Willett WC. Rosner BA. Egan KM. Faberowski N. Hankinson SE. Dietary fat consumption and primary open-angle glaucoma. Am J Clin Nutr. 2004;79:755-764.

124 Kant AK GB. Schatzkin A. Dietary patterns predict mortality in a national cohort: the National Health Interview Surveys. 1987 and 1992. J Nutr. 2004;134:1793-1799.

125 Kawamoto R TH. Oka Y. Kodama A. Ohtsuka N. Kamitani A. Association between uric acid and carotid atherosclerosis in elderly persons. Intern Med. 2005;44:787-793.

126 Keinan-Boker L vDSY. Grobbee DE. Peeters PH. Am J Clin Nutr. . Dietary phytoestrogens and breast cancer risk. 2004;79:282-288.

127 Khan MM HM. Kobayashi K. Sakauchi F. Yamashita T. Ahmed MF. Hossain MD. Quamruzzaman Q. Mori M. Levels of blood and urine chemicals associated with longer duration of having arsenicosis in Bangladesh. Int $J$ Environ Health Res. 2005;15:289-301.

128 Khaw KT BS. Welch A. Luben R. O'Brien E. Wareham N. Day N. Blood pressure and urinary sodium in men and women: the Norfolk Cohort of the European Prospective Investigation into Cancer (EPIC-Norfolk). Am J Clin Nutr. 2004;80:1397-1403.

129 Kiechl S SG. Wenning G. Redlich K. Oberhollenzer M. Mayr A. Santer P. Smolen J. Poewe W. Willeit J. Osteoprotegerin is a risk factor for progressive atherosclerosis and cardiovascular disease. Circulation. 2004;109:2175-2180. 
130 Kilkkinen A VJ. Vartiainen E. Sankila R. Virtanen MJ. Adlercreutz H. Pietinen P. Serum enterolactone concentration is not associated with breast cancer risk in a nested case-control study. Int J Cancer. 2004;108:277-280.

131 Kim MK SS. Sasazuki S. Tsugane S; Japan Public Health Center-based Prospective Study Group. Prospective study of three major dietary patterns and risk of gastric cancer in Japan. Int J Cancer. 2004;110:435-442.

132 Kim MK SS. Otani T. Tsugane S; Japan Public Health Center-based Prospective Study Group. Dietary patterns and subsequent colorectal cancer risk by subsite: a prospective cohort study. Int J Cancer. 2005;115(5):790-798

133 Kitamura A IH. Imano H. Ohira T. Okada T. Sato S. Kiyama M. Tanigawa T. Yamagishi K. Shimamoto T. Carotid intima-media thickness and plaque characteristics as a risk factor for stroke in Japanese elderly men. Stroke. 2004;35:2788-2794.

134 Klein R KB. Tomany SC. Wong TY. The relation of retinal microvascular characteristics to age-related eye disease: the Beaver Dam eye study. Am J Ophthalmol. 2004;137:435-444.

135 Knekt P RJ. Pereira MA. O'Reilly EJ. Augustsson K. Fraser GE. Goldbourt U. Heitmann BL. Hallmans G. Liu S. Pietinen P. Spiegelman D. Stevens J. Virtamo J. Willett WC. Rimm EB. Ascherio A. Antioxidant vitamins and coronary heart disease risk: a pooled analysis of 9 cohorts. Am J Clin Nutr. 2004;80:1508-1520.

136 Kobayashi M TY. Otani T. Hanaoka T. Sobue T. Tsugane S; JPHC Study Group. Fish. long-chain n-3 polyunsaturated fatty acids. and risk of colorectal cancer in middle-aged Japanese: the JPHC study. Nutr Cancer. 2004;49:32-40.

137 Koenig W TD. Brenner H. Rothenbacher D. Plasma concentrations of cystatin $\mathrm{C}$ in patients with coronary heart disease and risk for secondary cardiovascular events: more than simply a marker of glomerular filtration rate. Clin Chem. 2005;51:321-327.

138 Kojima M WK. Tokudome S. Suzuki K. Tamakoshi K. Watanabe Y. Kawado M. Hashimoto S. Hayakawa N. Ozasa K. Toyoshima H. Suzuki S. Ito Y. Tamakoshi A; JACC Study Group. Serum levels of polyunsaturated fatty acids and risk of colorectal cancer: a prospective study. Am J Epidemiol. 2005;161(5):462-471.

139 Koren-Morag N TD. Goldbourt U. White blood cell count and the incidence of ischemic stroke in coronary heart disease patients. Am J Med. 2005;118:10041009.

140 Koushik A HD. Spiegelman D. Anderson KE. Arslan AA. Beeson WL. van den Brandt PA. Buring JE. Cerhan JR. Colditz GA. Fraser GE. Freudenheim JL. Genkinger JM. Goldbohm RA. Hankinson SE. Koenig KL. Larsson SC. Leitzmann M. McCullough ML. Miller AB. Patel A. Rohan TE. Schatzkin A. Smit E. Willett WC. Wolk A. Zhang SM. Smith-Warner SA. Fruits and vegetables and ovarian cancer risk in a pooled analysis of 12 cohort studies. Cancer Epidemiol Biomarkers Prev. 2005;14:2160-2167.

141 Kragelund C GB. Kober L. Hildebrandt P. Steffensen R. N-terminal pro-Btype natriuretic peptide and long-term mortality in stable coronary heart disease. $N$ Engl J Med. 2005;352:666-675.

142 Krause N RR. Ragland DR. Syme SL. Physical workload. ergonomic problems. and incidence of low back injury: a 7.5-year prospective study of San Francisco transit operators. Am J Ind Med. 2004;46:570-585. 
143 Kurl S LJ. Niskanen L. Rauramaa R. Tuomainen TP. Sivenius J. Salonen JT. Cardiac power during exercise and the risk of stroke in men. Stroke. 2005;36:820-824.

144 Kurth T HC. Sturmer T. Sesso HD. Glynn RJ. Buring JE. Gaziano JM. Analgesic use and risk of subsequent hypertension in apparently healthy men. Arch Intern Med. 2005;165:1903-1909.

145 Kurzius-Spencer M HM. Carla Lohman I. Martinez FD. Wright AL. Prenatal factors associated with the development of eczema in the first year of life. Pediatr Allergy Immunol. 2005;16:19-26.

146 Larsson SC BL. Wolk A. Magnesium intake in relation to risk of colorectal cancer in women. JAMA. 2005;293:86-89.

147 Larsson SC BL. Wolk A. High-fat dairy food and conjugated linoleic acid ntakes in relation to colorectal cancer incidence in the Swedish Mammography Cohort. Am J Clin Nutr. 2005;82:894-900.

148 Larsson SC GE. Wolk A. Dietary folate intake and incidence of ovarian cancer: the Swedish Mammography Cohort. J Natl Cancer Inst. 2004;96:396402.

149 Larsson SC GE. Wolk A. Vitamin B6 intake. alcohol consumption. and colorectal cancer: a longitudinal population-based cohort of women. Gastroenterology. 2005;128:1830-1837.

150 Larsson SC GE. Wolk A. A prospective study of dietary folate intake and risk of colorectal cancer: modification by caffeine intake and cigarette smoking. Cancer Epidemiol Biomarkers Prev. 2005;14:740-743.

151 Leitzmann MF SM. Michaud DS. Augustsson K. Colditz GC. Willett WC. Giovannucci EL. Dietary intake of n-3 and n-6 fatty acids and the risk of prostate cancer. Am J Clin Nutr. 2004;80:204-216.

152 Leosdottir M NP. Nilsson JA. Mansson H. Berglund G. Dietary fat intake and early mortality patterns-data from The Malmo Diet and Cancer Study. J Intern Med. 2005;258:153-165.

153 Leung H WJ. Rochtchina E. Wong TY. Klein R. Mitchell P. Impact of current and past blood pressure on retinal arteriolar diameter in an older population. $J$ Hypertens. 2004;22:1543-1549.

154 Li G SJ. Kukull WA. Peskind ER. Tsuang DW. Breitner JC. McCormick W. Bowen JD. Teri L. Schellenberg GD. Larson EB. Serum cholesterol and risk of Alzheimer disease: a community-based cohort study. Neurology. 2005;65:1045-1050.

155 Lim S LH. Kimm KC. Park C. Shin C. Cho NH. C-reactive protein level as an independent risk factor of metabolic syndrome in the Korean population CRP as risk factor of metabolic syndrome. Diabetes Res Clin Pract. 2005;70:126133.

156 Lin J ZS. Cook NR. Lee IM. Buring JE. Dietary fat and fatty acids and risk of colorectal cancer in women. Am J Epidemiol. 2004;160(10):1011-1022.

157 Lin J ZS. Cook NR. Rexrode KM. Liu S. Manson JE. Lee IM. Buring JE. Dietary intakes of fruit. vegetables. and fiber. and risk of colorectal cancer in a prospective cohort of women (United States). Cancer Causes Control. 2005;16:225-233.

158 Lin J ZS. Cook NR. Manson JE. Lee IM. Buring JE. Intakes of calcium and vitamin D and risk of colorectal cancer in women. Am J Epidemiol. 2005;161:755-764. 
159 Lin Y TA. Kikuchi S. Yagyu K. Obata Y. Ishibashi T. Kawamura T. Inaba Y. Kurosawa M. Motohashi Y. Ohno Y. Serum insulin-like growth factor-I. insulin-like growth factor binding protein-3. and the risk of pancreatic cancer death. Int J Cancer. 2004;110:584-588.

160 Loucks EB BL. Gruenewald TL. Seeman TE. Social integration is associated with fibrinogen concentration in elderly men. Psychosom Med. 2005;67:353358.

161 Louis GM WJ. Whitcomb BW. Sperrazza R. Schisterman EF. Lobdell DT. Crickard K. Greizerstein H. Kostyniak PJ. Environmental PCB exposure and risk of endometriosis. Hum Reprod. 2004;20:279-285.

162 Lu M CE. Taylor A. Hankinson SE. Willett WC. Jacques PF. Prospective study of dietary fat and risk of cataract extraction among US women. Am $J$ Epidemiol. 2005;161:948-959.

163 Lu M TA. Chylack LT Jr. Rogers G. Hankinson SE. Willett WC. Jacques PF. Dietary fat intake and early age-related lens opacities. Am J Clin Nutr. 2005;81(4):773-779.

164 Luchsinger JA TM. Shea S. Mayeux R. Hyperinsulinemia and risk of Alzheimer disease. Neurology. 2004;63:1187-1192.

165 Luchsinger JA TM. Shea S. Miller J. Green R. Mayeux R. Plasma homocysteine levels and risk of Alzheimer disease. Neurology. 2004;62:19721976.

166 Luchtenborg M WM. de Goeij AF. Wark PA. Brink M. Roemen GM. Lentjes MH. de Bruine AP. Goldbohm RA. van 't Veer P. van den Brandt PA. Meat and Fish Consumption. APCGene Mutations and hMLH1 Expression in Colon and Rectal Cancer: a Prospective Cohort Study (The Netherlands). Cancer Causes Control. 2005;19:1041-54.

167 Lukanova A. Bjor O. Kaaks R. et al. Body mass index and cancer: results from the Northern Sweden Health and Disease Cohort. Int J Cancer. Jan 15 2006;118:458-466.

168 Lukanova A. Zeleniuch-Jacquotte A. Lundin E. et al. Prediagnostic levels of C-peptide. IGF-I. IGFBP -1. -2 and -3 and risk of endometrial cancer. Int J Cancer. Jan 10 2004;108:262-268.

169 Lukanova A LE. Micheli A. Arslan A. Ferrari P. Rinaldi S. Krogh V. Lenner P. Shore RE. Biessy C. Muti P. Riboli E. Koenig KL. Levitz M. Stattin P. Berrino F. Hallmans G. Kaaks R. Toniolo P. Zeleniuch-Jacquotte A. Circulating levels of sex steroid hormones and risk of endometrial cancer in postmenopausal women. Int J Cancer. 2004;108:425-432.

170 Lundblad D DB. Rautio A. Roder ME. Eliasson M. Low level of tissue plasminogen activator activity in non-diabetic patients with a first myocardial infarction. J Intern Med. 2005;258:13-20.

171 Luo ZC KW. Wilkins R. Liston RM. Mohamed J. Kramer MS; British Columbia Vital Statistics Agency. Disparities in birth outcomes by neighborhood income: temporal trends in rural and urban areas. british columbia. Epidemiology. 2004;15:679-686.

172 MacInnis RJ ED. Hopper JL. Haydon AM. Gertig DM. Giles GG. Body size and composition and colon cancer risk in men. Cancer Epidemiol Biomarkers Prev. 2004;13:553-559.

173 Madsen TE MJ. Carlquist JF. Horne BD. Bair TL. Jackson JD. Lappe JM. Pearson RR. Anderson JL. Serum uric acid independently predicts mortality in 
patients with significant. angiographically defined coronary disease. Am J Nephrol. 2005;25:45-49.

174 Mahabir S LM. Virtanen MJ. Virtamo J. Pietinen P. Albanes D. Taylor PR. Prospective study of alcohol drinking and renal cell cancer risk in a cohort of finnish male smokers. Cancer Epidemiol Biomarkers Prev. 2005;14:170-175.

175 Mai V KA. Flood A. Lacey JV Jr. Schairer C. Schatzkin A. Diet quality and subsequent cancer incidence and mortality in a prospective cohort of women. Int J Epidemiol. 2005;34:54-60.

176 Mallamaci F BG. Seminara G. Rapisarda F. Fatuzzo P. Candela V. Scudo P. Spoto B. Testa A. Tripepi G. Tech S. Zoccali C. Hyperhomocysteinemia and arteriovenous fistula thrombosis in hemodialysis patients. Am J Kidney Dis. 2005;45:702-707.

177 Mangtani P BE. Kovats S. Ng ES. Roberts JA. Fletcher A. Inequalities in influenza vaccine uptake among people aged over 74 years in Britain. Prev Med. . 2005;41:545-553.

178 Manjer J JR. Lenner P. Smoking is associated with postmenopausal breast cancer in women with high levels of estrogens. Int J Cancer. 2004;112:324328.

179 Mannisto S DL. Balder HF. Virtanen MJ. Krogh V. Khani BR. Berrino F. van den Brandt PA. Hartman AM. Pietinen P. Tan F. Wolk A. Goldbohm RA. Dietary patterns and breast cancer risk: results from three cohort studies in the DIETSCAN project. Cancer Causes Control. 2005;16:725-733.

180 Mannisto S S-WS. Spiegelman D. Albanes D. Anderson K. van den Brandt PA. Cerhan JR. Colditz G. Feskanich D. Freudenheim JL. Giovannucci E. Goldbohm RA. Graham S. Miller AB. Rohan TE. Virtamo J. Willett WC. Hunter DJ. Dietary carotenoids and risk of lung cancer in a pooled analysis of seven cohort studies. Cancer Epidemiol Biomarkers Prev. 2004;13(1):40-48.

181 Margolis KL MJ. Greenland P. Rodabough RJ. Bray PF. Safford M. Grimm RH Jr. Howard BV. Assaf AR. Prentice R; Women's Health Initiative Research Group. Leukocyte count as a predictor of cardiovascular events and mortality in postmenopausal women: the Women's Health Initiative Observational Study. Arch Intern Med. 2005;165:500-508.

182 Maru S vdSY. Gimbrere CH. Grobbee DE. Peeters PH. Body mass index and short-term weight change in relation to mortality in Dutch women after age 50 y. Am J Clin Nutr. 2004;80:231-236.

183 Massy ZA FC. Borderie D. Tuppin P. Nguyen-Khoa T. Benoit MO. Jacquot C. Buisson C. Drueke TB. Ekindjian OG. Lacour B. Iliou MC. Increased pasma S-nitrosothiol concentrations predict cardiovascular outcomes among patients with end-stage renal disease: a prospective study. J Am Soc Nephrol. 2004;15:470-476.

184 Mattisson I WE. Johansson U. Gullberg B. Olsson H. Berglund G. Intakes of plant foods. fibre and fat and risk of breast cancer--a prospective study in the Malmo Diet and Cancer cohort. Br J Cancer. 2004;90:122-127.

185 Mayne ST. Cartmel B. Lin H. Zheng T. Goodwin WJ. Jr. Low plasma lycopene concentration is associated with increased mortality in a cohort of patients with prior oral. pharynx or larynx cancers. J Am Coll Nutr. Feb 2004;23:34-42.

186 McKeown NM MJ. Liu S. Saltzman E. Wilson PW. Jacques PF. Carbohydrate nutrition. insulin resistance. and the prevalence of the metabolic syndrome in the Framingham Offspring Cohort. Diabetes Care. 2004;27:538-546. 
187 Mehling WE KN. Are difficulties perceiving and expressing emotions associated with low-back pain? The relationship between lack of emotional awareness (alexithymia) and 12-month prevalence of low-back pain in 1180 urban public transit operators. J Psychosom Res. 2005;58:73-81.

188 Meier C NT. Center JR. Seibel MJ. Eisman JA. Bone resorption and osteoporotic fractures in elderly men: the dubbo osteoporosis epidemiology study. J Bone Miner Res. 2005;20:579-587.

189 Merkin SS CJ. Roux AV. Taylor HA. Powe NR. Area socioeconomic status and progressive CKD: the Atherosclerosis Risk in Communities (ARIC) Study. Am J Kidney Dis. 2005;46:203-213.

190 Merlino LA CJ. Mikuls TR. Cerhan JR. Criswell LA. Saag KG; Iowa Women's Health Study. Vitamin D intake is inversely associated with rheumatoid arthritis: results from the Iowa Women's Health Study. Arthritis Rheum. 2004;50:72-77.

191 Messer LC. Dole N. Kaufman JS. Savitz DA. Pregnancy Intendedness. Maternal Psychosocial Factors and Preterm Birth. Matern Child Health J. 2005:1-10.

192 Meyerhardt JA TJ. Niedzwiecki D. Hollis DR. Schrag D. Ayanian JZ. O'Connell MJ. Weeks JC. Mayer RJ. Willett CG. MacDonald JS. Benson AB 3rd. Fuchs CS. Impact of hospital procedure volume on surgical operation and long-term outcomes in high-risk curatively resected rectal cancer: findings from the Intergroup 0114 Study. J Clin Oncol. . 2004;22:166-174.

193 Michaud DS FC. Liu S. Willett WC. Colditz GA. Giovannucci E. Dietary glycemic load. carbohydrate. sugar. and colorectal cancer risk in men and women. Cancer Epidemiol Biomarkers Prev. 2005;14:138-147.

194 Michaud DS SH. Wu K. Hu F. Giovannucci E. Willett WC. Colditz GA. Fuchs CS. Dietary patterns and pancreatic cancer risk in men and women. $J$ Natl Cancer Inst. 2005;97:518-524.

195 Michaud DS WM. Cantor KP. Taylor PR. Virtamo J. Albanes D. Arsenic concentrations in prediagnostic toenails and the risk of bladder cancer in a cohort study of male smokers. Am J Epidemiol. 2004;160:853-859.

196 Micheli A MP. Secreto G. Krogh V. Meneghini E. Venturelli E. Sieri S. Pala V. Berrino F. Endogenous sex hormones and subsequent breast cancer in premenopausal women. Int J Cancer. 2004;112:312-318.

197 Mielke MM. Zandi PP. Sjogren M. et al. High total cholesterol levels in late life associated with a reduced risk of dementia. Neurology. May 24 2005;64:1689-1695.

198 Miller AB AH. Bueno-de-Mesquita B. Boshuizen HC. Agudo A. Berrino F. Gram IT. Janson L. Linseisen J. Overvad K. Rasmuson T. Vineis P. Lukanova A. Allen N. Amiano P. Barricarte A. Berglund G. Boeing H. Clavel-Chapelon F. Day NE. Hallmans G. Lund E. Martinez C. Navarro C. Palli D. Panico S. Peeters PH. Quiros JR. Tjonneland A. Tumino R. Trichopoulou A.

Trichopoulos D. Slimani N. Riboli E. Fruits and vegetables and lung cancer: Findings from the European Prospective Investigation into Cancer and Nutrition. Int J Cancer. 2004;108:269-272.

199 Mishra GD BK. Dobson AJ. Byles JE. Do socioeconomic gradients in women's health widen over time and with age? Soc Sci Med. 2004;58:15851595.

200 Molyneux A LS. Antoniak M. Browne W. McNeill A. Godfrey C. Madeley R. Britton J. Prospective study of the effect of exposure to other smokers in high 
school tutor groups on the risk of incident smoking in adolescence. Am $J$ Epidemiol. 2004;159:127-132.

201 Mommers M SL. Goldbohm RA. van den Brandt PA. Consumption of vegetables and fruits and risk of ovarian carcinoma. Cancer. 2005;104:15121519.

202 Montonen J. Knekt P. Harkanen T. et al. Dietary patterns and the incidence of type 2 diabetes. Am J Epidemiol. Feb 1 2005;161:219-227.

203 Montonen J. Knekt P. Jarvinen R. Reunanen A. Dietary antioxidant intake and 7risk of type 2 diabetes. Diabetes Care. Feb 2004;27:362-366.

204 Montonen J JR. Heliovaara M. Reunanen A. Aromaa A. Knekt P. Food consumption and the incidence of type II diabetes mellitus. Eur J Clin Nutr. 2005;59:441-448.

205 Morange PE SC. Alessi MC. Luc G. Arveiler D. Ferrieres J. Amouyel P. Evans A. Ducimetiere P. Juhan-Vague I; PRIME Study Group. Endothelial cell markers and the risk of coronary heart disease: the Prospective Epidemiological Study of Myocardial Infarction (PRIME) study. Circulation. 2004;109:1343-1348Circulation.

206 Mozaffarian D BC. Lemaitre RN. Burke GL. Siscovick DS. Fish intake and risk of incident heart failure. J Am Coll Cardiol. 2005;45:2015-2021.

207 Mucci LA AH. Wolk A. Prospective study of dietary acrylamide and risk of colorectal cancer among women. Int J Cancer. 2005;118:169-173.

208 Munger KL ZS. O'Reilly E. Hernan MA. Olek MJ. Willett WC. Ascherio A. Vitamin D intake and incidence of multiple sclerosis. Neurology. 2004;62:6065.

209 Myint PK. Luben RN. Surtees PG. et al. Relation Between Self-Reported Physical Functional Health and Chronic Disease Mortality in Men And Women in the European Prospective Investigation Into Cancer (EPICNorfolk): A Prospective Population Study. Ann Epidemiol. 2005.

210 Nagata C TN. Shimizu N. Shimizu H. Sodium intake and risk of death from stroke in Japanese men and women. Stroke. 2004;35:1543-1547.

211 Nagel G LJ. Dietary intake of fatty acids. antioxidants and selected food groups and asthma in adults. Eur J Clin Nutr. 2005;59:8-15.

212 Nair GV CL. Vittinghoff E. Herrington DM; Heart and Estrogen/Progestin Replacement Study Investigators. Pulse pressure and cardiovascular events in postmenopausal women with coronary heart disease. Chest. 2005;127:14981506.

213 Nakanishi N SK. Arch Intern Med. . Daily life activity and the risk of developing hypertension in middle-aged Japanese men. 2005;165(2):214-220.

214 Navas-Acien A SE. Sharrett AR. Calderon-Aranda E. Silbergeld E. Guallar E. Lead. cadmium. smoking. and increased risk of peripheral arterial disease. Circulation. 2004;109):3196-3201.

214 Ness AR MM. Frankel S. Smith GD. Frobisher C. Leary SD. Emmett PM. Gunnell D. Diet in childhood and adult cardiovascular and all cause mortality: the Boyd Orr cohort. Heart. 2005;91:894-898.

216 Nguyen L CT. Vanichseni S. McNicholl JM. Mock PA. Nelson R. Hodge TW. van Griensven F. Choopanya K. Mastro TD. Tappero JW. Hu DJ. Frequent human leukocyte antigen class I alleles are associated with higher viral load among HIV type 1 seroconverters in Thailand. J Acquir Immune Defic Syndr. . 2004;37:1318-1323. 
217 Ninomiya T KY. Kubo M. Tanizaki Y. Tanaka K. Okubo K. Nakamura H. Hata J. Oishi Y. Kato I. Hirakata H. Iida M. Hyperhomocysteinemia and the development of chronic kidney disease in a general population: the Hisayama study. Am J Kidney Dis. . 2004;44:437-445.

217 Nothlings U WL. Murphy SP. Hankin JH. Henderson BE. Kolonel LN. Meat and fat intake as risk factors for pancreatic cancer: the multiethnic cohort study. J Natl Cancer Inst. 2005;97:1458-1465.

219 Oei HH vdMI. Hofman A. Koudstaal PJ. Stijnen T. Breteler MM. Witteman JC. Lipoprotein-associated phospholipase A2 activity is associated with risk of coronary heart disease and ischemic stroke: the Rotterdam Study. Circulation. 2005;111:570-575.

220 Oh JC WW. Tortolero-Luna G. Broaddus R. Gershenson DM. Burke TW. Schmandt R. Lu KH. Increased plasma levels of insulin-like growth factor 2 and insulin-like growth factor binding protein 3 are associated with endometrial cancer risk. Cancer Epidemiol Biomarkers Prev. 2004;13:748752.

221 Oh K WW. Fuchs CS. Giovannucci EL. Glycemic index. glycemic load. and carbohydrate intake in relation to risk of distal colorectal adenoma in women. Cancer Epidemiol Biomarkers Prev. 2004;13:1192-1198.

222 Okamura T HT. Kadowaki T. Kita Y. Okayama A. Elliott P. Ueshima H; NIPPONDATA80 Research Group. Resting heart rate and cause-specific death in a 16.5-year cohort study of the Japanese general population. Am Heart J. 2004;147:1024-1032.

223 Olijhoek JK KJ. de Jaegere PP. Verhaar MC. Grobbee DE. van der Graaf Y. Visseren FL; SMART Study Group. Presence of the metabolic syndrome does not impair coronary collateral vessel formation in patients with documented coronary artery disease. Diabetes Care. 2005;28:683-689.

224 Olivot JM LJ. Aiach M. Amarenco P; GENIC Investigators. Soluble thrombomodulin and brain infarction: case-control and prospective study. Stroke. 2004;35:1946-1951.

225 Olson MB KD. Kelsey SF. Pepine CJ. Sopko G. Handberg E. Rogers WJ. Gierach GL. McClure CK. Merz CN; WISE Study Group. Hostility scores are associated with increased risk of cardiovascular events in women undergoing coronary angiography: a report from the NHLBI-Sponsored WISE Study. Psychosom Med. 2005;67:546-562.

226 Page JH CG. Rifai N. Barbieri RL. Willett WC. Hankinson SE. Plasma adrenal androgens and risk of breast cancer in premenopausal women. Cancer Epidemiol Biomarkers Prev. 2004;13:1032-1036.

227 Panichi V MU. Taccola D. Migliori M. Rizza GM. Consani C. Bertini A. Sposini S. Perez-Garcia R. Rindi P. Palla R. Tetta C. Interleukin-6 is a stronger predictor of total and cardiovascular mortality than C-reactive protein in haemodialysis patients. Nephrol Dial Transplant. 2004;19:1154-1160.

228 Pankow JS DB. Schmidt MI. Ballantyne CM. Couper DJ. Hoogeveen RC. Golden SH; Atherosclerosis Risk in Communities Study. Fasting plasma free fatty acids and risk of type 2 diabetes: the atherosclerosis risk in communities study. Diabetes Care. 2004;27:77-82.

229 Park HA LJ. Kuller LH. Relationship between premenopausal dietary intake and postmenopausal subclinical atherosclerosis. Atherosclerosis. 2005.

230 Pelfrene E VP. Moreau M. Mak RP. Kornitzer M. De Backer G. Use of benzodiazepine drugs and perceived job stress in a cohort of working men and 
women in Belgium. Results from the BELSTRESS-study. Soc Sci Med. 2004;59:433-442.

231 Pell JP. Corstorphine M. McConnachie A. et al. Post-discharge survival following pre-hospital cardiopulmonary arrest due to cardiac aetiology: temporal trends and impact of changes in clinical management. Eur Heart J. Feb 2006;27:406-412.

232 Pell JP SG. Walsh D. Pregnancy complications and subsequent maternal cerebrovascular events: a retrospective cohort study of 119.668 births. Am J Epidemiol. 2004;159:336-342.

233 Pena de la Vega L MR. Benda MM. Grill DE. Johnson MG. McCarthy JT. McBane RD 2nd. Association of heparin-dependent antibodies and adverse outcomes in hemodialysis patients: a population-based study. Mayo Clin Proc. 2005;80:995-1000.

234 Phipatanakul W CJ. Sredl DL. Weiss ST. Gold DR. Mouse exposure and wheeze in the first year of life. Ann Allergy Asthma Immunol. 2005;94:593599.

235 Platek M KV. Micheli A. Browne R. Meneghini E. Sieri S. Schunemann HJ. Pala V. Barba M. Wilding GE. Berrino F. Muti P. Serum fructosamine and subsequent breast cancer risk: a nested case-control study in the ORDET prospective cohort study. Cancer Epidemiol Biomarkers Prev. 2005;14:271274.

236 Polychronopoulos E PD. Polystipioti A.. Diet. lifestyle factors and hypercholesterolemia in elderly men and women from Cyprus. Lipids Health Dis. 2005;4:17.

237 Pussinen PJ AG. Tuomilehto J. Asikainen S. Jousilahti P. High serum antibody levels to Porphyromonas gingivalis predict myocardial infarction. Eur J Cardiovasc Prev Rehabil. 2004;11:408-411.

238 Qiu C FL. Karp A. Winblad B. Bellander T. Occupational exposure to electromagnetic fields and risk of Alzheimer's disease. Epidemiology. 2004;15:687-694.

239 Qiu C ST. Luthy DA. Williams MA. A prospective study of maternal serum C-reactive protein (CRP) concentrations and risk of gestational diabetes mellitus. Paediatr Perinat Epidemiol. . 2004;18:377-384.

240 Raaschou-Nielsen O PM. Leblanc A. Dumas P. Philippe Weber J. Olsen A. Tjonneland A. Overvad K. Olsen JH. Adipose organochlorine concentrations and risk of breast cancer among postmenopausal Danish women. Cancer Epidemiol Biomarkers Prev. 2005;14:67-74.

241 Rajala U LM. Paivansalo M. Suramo I. Keinanen-Kiukaanniemi S. Blood pressure and atherosclerotic plaques in carotid. aortic and femoral arteries in elderly Finns with diabetes mellitus or impaired glucose tolerance. J Hum Hypertens. . 2005;19:85-91.

242 Rajpathak S RE. Morris JS. Hu F. Toenail selenium and cardiovascular disease in men with diabetes. J Am Coll Nutr. 2005;24(4):250-256.

243 Ramos MI AL. Haan MN. Green R. Miller JW. Plasma folate concentrations are associated with depressive symptoms in elderly Latina women despite folic acid fortification. Am J Clin Nutr. . 2004;80:1024-1028.

244 Rashidkhani B AA. Lindblad P. Wolk A. Major dietary patterns and risk of renal cell carcinoma in a prospective cohort of Swedish women. $J$ Nutr. . 2005;135:1757-1762. 
245 Ray JG WP. Vermeulen MJ. Meier C. Cole DE. Greater maternal weight and the ongoing risk of neural tube defects after folic acid flour fortification. Obstet Gynecol. 2005;105:261-265.

246 Reijman M HJ. Bierma-Zeinstra SM. Koes BW. Christgau S. Christiansen C. Uitterlinden AG. Pols HA. A new marker for osteoarthritis: cross-sectional and longitudinal approach. Arthritis Rheum. 2004;50:2471-2478.

247 Ribeiro JC GS. Oliveira J. Andersen LB. Duarte JA. Mota J. Body fatness and clustering of cardiovascular disease risk factors in Portuguese children and adolescents. Am J Hum Biol. 2004;16:556-562.

248 Richardson DB WS. Lorey F. Hertz-Picciotto I. Adult hemoglobin levels at birth and risk of sudden infant death syndrome. Arch Pediatr Adolesc Med. 2004;158:366-371.

249 Ridker PM RN. Cook NR. Bradwin G. Buring JE. Non-HDL cholesterol. apolipoproteins A-I and B100. standard lipid measures. lipid ratios. and CRP as risk factors for cardiovascular disease in women. JAMA. 2005;294:326-333.

250 Rinaldi S. Kaaks R. Zeleniuch-Jacquotte A. et al. Insulin-like growth factor-I. IGF binding protein-3. and breast cancer in young women: a comparison of risk estimates using different peptide assays. Cancer Epidemiol Biomarkers Prev. Jan 2005;14:48-52.

251 Rock CL FS. Natarajan L. Thomson CA. Bardwell WA. Newman VA. Hollenbach KA. Jones L. Caan BJ. Pierce JP. Plasma carotenoids and recurrence-free survival in women with a history of breast cancer. $J$ Clin Oncol. 2005;23:6631-6638.

252 Rosengren A WL. Orth-Gomer K. Coronary disease in relation to social support and social class in Swedish men. A 15 year follow-up in the study of men born in 1933. Eur Heart J. 2004;25:56-63.

253 Rosenthal AD JF. Shu XO. Yang G. Elasy TA. Chow WH. Ji BT. Xu HX. Li Q. Gao YT. Zheng W. Body fat distribution and risk of diabetes among Chinese women. Int J Obes Relat Metab Disord. 2004;28:594-599.

254 Rossi R CE. Nuzzo A. Cioni E. Origliani G. Modena MG. Flow-mediated vasodilation and the risk of developing hypertension in healthy postmenopausal women. J Am Coll Cardiol. 2004;44:1636-1640.

255 Rossi R CE. Nuzzo A. Origliani G. Modena MG. Endothelial-dependent vasodilation and incidence of type 2 diabetes in a population of healthy postmenopausal women. Diabetes Care. 2005;28:702-707.

256 Rouillier P B-RM. Bertrais S. Arnault N. Daudin JJ. Bacro JN. Hercberg S. Alcohol and atherosclerotic vascular disease risk factors in French men: relationships are linear. J-shaped. and U-shaped. Alcohol Clin Exp Res. 2005;29:84-88.

257 Rumbold AR MF. Crowther CA. Dietary intake of vitamin C and vitamin E and the development of hypertensive disorders of pregnancy. Eur J Obstet Gynecol Reprod Biol. 2005;119:67-71.

258 Rusiecki JA DRA. Lee WJ. Dosemeci M. Lubin JH. Hoppin JA. Blair A. Alavanja MC. Cancer incidence among pesticide applicators exposed to atrazine in the Agricultural Health Study. J Natl Cancer Inst. 2004;96:13751382.

259 Rutter MK MJ. Sullivan LM. D'Agostino RB Sr. Wilson PW. C-reactive protein. the 3037metabolic syndrome. and prediction of cardiovascular events in the Framingham Offspring Study. Circulation. 2004;110:380-385. 
260 Salvarani C CL. Macchioni P. Consonni D. Cantini F. Bajocchi G. Pipitone N. Catanoso MG. Boiardi L. Risk factors for visual loss in an Italian populationbased cohort of patients with giant cell arteritis. Arthritis Rheum. 2005;53:293297.

261 Samelson EJ KD. Broe KE. Zhang Y. Cupples LA. Hannan MT. Wilson PW. Levy D. Williams SA. Vaccarino V. Metacarpal cortical area and risk of coronary heart disease: the Framingham Study. Am J Epidemiol. 2004;159:589-595.

262 Sanchez PL MJ. Pabon P. Martin F. Piedra I. Palacios IF. Martin-Luengo C. Prognostic relations between inflammatory markers and mortality in diabetic patients with non-ST elevation acute coronary syndrome. Heart. 2004;90:264269.

263 Sato Y TY. Nakaya N. Ogawa K. Kurashima K. Kuriyama S. Hozawa A. Nishino Y. Shibuya D. Tsuji I. Fruit and vegetable consumption and risk of colorectal cancer in Japan: The Miyagi Cohort Study. Public Health Nutr. 2005;8:309-314.

264 Saw SM TS. Fung D. Chia KS. Koh D. Tan DT. Stone RA. IQ and the association with myopia in children. Invest Ophthalmol Vis Sci. 2004;45:24932498.

265 Schaumberg DA LS. Seddon JM. Willett WC. Hankinson SE. Dietary glycemic load and risk of age-related cataract. Am J Clin Nutr. 2004;80:489495.

266 Schernhammer ES HJ. Pollak MN. Hankinson SE. Circulating levels of insulin-like growth factors. their binding proteins. and breast cancer risk. Cancer Epidemiol Biomarkers Prev. 2005;14:699-704.

267 Schernhammer ES HS. Urinary melatonin levels and breast cancer risk. J Natl Cancer Inst. 2005;97:1084-1087.

268 Schillinger M EM. Sabeti S. Mlekusch W. Amighi J. Handler S. Quehenberger P. Kalifeh N. Wagner O. Minar E. High plasma heparin cofactor II activity protects from restenosis after femoropopliteal stenting. Thromb Haemost. 2004;95:1108-1113.

269 Schnabel R RH. Lackner KJ. Lubos E. Bickel C. Meyer J. Munzel T. Cambien F. Tiret L. Blankenberg S; AtheroGene Investigators. Analysis of N-terminalpro-brain natriuretic peptide and C-reactive protein for risk stratification in stable and unstable coronary artery disease: results from the AtheroGene study. Eur Heart J. 2005;26:241-249.

270 Schoen RE WJ. Kuller LH. Thaete FL. Evans RW. Hayes RB. Rosen CJ. Insulin-like growth factor-I and insulin are associated with the presence and advancement of adenomatous polyps. Gastroenterology. 2005;129:464-475.

271 Schuiling WJ dWA. Dennesen PJ. Algra A. Rinkel GJ. The simplified acute physiology score to predict outcome in patients with subarachnoid hemorrhage. Neurosurgery. 2005;57:230-236.

272 Schulze MB HK. Manson JE. Willett WC. Meigs JB. Weikert C. Heidemann C. Colditz GA. Hu FB. Dietary pattern. inflammation. and incidence of type 2 diabetes in women. Am J Clin Nutr. 2005;82:675-684.

273 Schulze MB LS. Rimm EB. Manson JE. Willett WC. Hu FB. Glycemic index. glycemic load. and dietary fiber intake and incidence of type 2 diabetes in younger and middle-aged women. Am J Clin Nutr. 2004;80:348-356. 
274 Schulze MB RE. Li T. Rifai N. Stampfer MJ. Hu FB. C-reactive protein and incident cardiovascular events among men with diabetes. Diabetes Care. 2004;27:889-894.

275 Schulze MB SC. Rifai N. Cohen RM. Sparrow J. Hu FB. Manson JE. Hyperproinsulinaemia and risk of Type 2 diabetes mellitus in women. Diabet Med. 2005;22:1178-1184.

276 Schulze MB SI. Manson JE. Li T. Rifai N. Jiang R. Hu FB. Joint role of nonHDL cholesterol and glycated haemoglobin in predicting future coronary heart disease events among women with type 2 diabetes. Diabetologia. 2004;47:2129-2136.

277 Schupf N CR. Luchsinger J. Tang MX. Lee JH. Mayeux R. Relationship between plasma lipids and all-cause mortality in nondemented elderly. $J$ Am Geriatr Soc. 2005;53:219-226.

278 Seddon JM GS. Rosner B. Rifai N. Progression of age-related macular degeneration: prospective assessment of C-reactive protein. interleukin 6. and other cardiovascular biomarkers. Arch Ophthalmol. 2005;123:774-782.

279 Selvin E CJ. Golden SH. Brancati FL. Folsom AR. Steffes MW. Glycemic control and coronary heart disease risk in persons with and without diabetes: the atherosclerosis risk in communities study. Arch Intern Med. 2005;165:1910-1916.

280 Selvin E CJ. Golden SH. Boland LL. Brancati FL. Steffes MW; Atherosclerosis risk in communities study. Glycemic control. atherosclerosis. and risk factors for cardiovascular disease in individuals with diabetes: the atherosclerosis risk in communities study. Diabetes Care. 2005;28:1965-1973.

281 Sesso HD BJ. Norkus EP. Gaziano JM. Plasma lycopene. other carotenoids. and retinol and the risk of cardiovascular disease in women. Am J Clin Nutr. 2004;79:47-53.

282 Sesso HD BJ. Zhang SM. Norkus EP. Gaziano JM. Dietary and plasma lycopene and the risk of breast cancer. Cancer Epidemiol Biomarkers Prev. 2005;14:1074-1081.

283 Shai I SM. Manson JE. Stampfer MJ. Rifai N. Hu FB. A prospective study of lipoprotein(a) and risk of coronary heart disease among women with type 2 diabetes. Diabetologia. 2005;48:1469-1476.

284 Shalak LF PJ. Jackson GL. Laptook AR. Depression at birth in term infants exposed to maternal chorioamnionitis: does neonatal fever play a role? $J$ Perinatol. . 2005;25:447-452.

285 Shankar A KB. Klein R. Relationship between white blood cell count and incident hypertension. Am J Hypertens. 2004;17:233-239.

286 Sheehan J PI. Reilly M. Salim A. Collins M. Twomey EM. Daly A. Loingsigh SN. Elwood P. Ben-Shlomo Y. Davey-Smith G. QT dispersion. QT maximum and risk of cardiac death in the Caerphilly Heart Study. Eur J Cardiovasc Prev Rehabil. 2004;11:63-68.

287 Shy BD RT. Becker LJ. Eisenberg MS. Time to intubation and survival in prehospital cardiac arrest. Prehosp Emerg Care. 2004;8:394-398.

288 Sieri S. Krogh V. Pala V. et al. Dietary patterns and risk of breast cancer in the ORDET cohort. Cancer Epidemiol Biomarkers Prev. Apr 2004;13(4):567-572.

289 Silvera SA JM. Howe GR. Miller AB. Rohan TE. Dietary carbohydrates and breast cancer risk: a prospective study of the roles of overall glycemic index and glycemic load. Int J Cancer. 2005;114:653-658. 
290 Silvera SA RT. Jain M. Terry PD. Howe GR. Miller AB. Glycemic index. glycemic load. and pancreatic cancer risk (Canada). Cancer Causes Control. 2005;16:431-436.

291 Sin DD WL. Man SF. The relationship between reduced lung function and ardiovascular mortality: a population-based study and a systematic review of the literature. Chest. 2005;127:1952-1959.

292 Singh JA ND. Fink HA. Nichol KL. Health-related quality of life predicts future health care utilization and mortality in veterans with self-reported physician-diagnosed arthritis: the veterans arthritis quality of life study. Semin Arthritis Rheum. . 2005;34:755-765.

293 Singh SM PL. Li C. He J. Vinden C. Rabeneck L. Association of socioeconomic status and receipt of colorectal cancer investigations: a population-based retrospective cohort study. CMAJ. 2004;171(5):461-465.

294 Skuladottir H TA. Overvad K. Stripp C. Christensen J. Raaschou-Nielsen O. Olsen JH. Does insufficient adjustment for smoking explain the preventive effects of fruit and vegetables on lung cancer? Lung Cancer. 2004;45:1-10.

295 Slinin Y FR. Collins AJ. Calcium. phosphorus. parathyroid hormone. and cardiovascular disease in hemodialysis patients: the USRDS waves 1. 3. and 4 study. J Am Soc Nephrol. 2005;16:1788-1793.

296 Smith W WJ. Wong TY. Rochtchina E. Klein R. Leeder SR. Mitchell P. Retinal arteriolar narrowing is associated with 5-year incident severe hypertension: the Blue Mountains Eye Study. Hypertension. 2004;44:442-447.

297 Song Y MJ. Buring JE. Liu S. Dietary magnesium intake in relation to plasma insulin levels and risk of type 2 diabetes in women. Diabetes Care. 2004;27:59-65.

298 Song Y MJ. Cook NR. Albert CM. Buring JE. Liu S. Dietary Magnesium Intake and Risk of Cardiovascular Disease Among Women. Am J Cardiol. 2005;96:1135-1141.

299 Sornay-Rendu E MF. Duboeuf F. Delmas PD. Rate of Forearm Bone Loss Is Associated With an Increased Risk of Fracture Independently of Bone Mass in Postmenopausal Women: The OFELY Study. J Bone Miner Res. . 2005;20:1929-1935.

300 Sornay-Rendu E MF. Garnero P. Duboeuf F. Delmas PD. Identification of Osteopenic Women at High Risk of Fracture: The OFELY Study. J Bone Miner Res. 2005;20:1813-1819.

301 Sprenkle MD ND. Nelson DB. Nichol KL. The Veterans Short Form 36 questionnaire is predictive of mortality and health-care utilization in a population of veterans with a self-reported diagnosis of asthma or COPD. Chest. 2004;126:81-89.

302 Stack AG MB. Molony DA. Survival differences between peritoneal dialysis and hemodialysis among "large" ESRD patients in the United States. Kidney Int. 2004;65(6):2398-2408.

303 Stattin P BA. Biessy C. Kaaks R. Hallmans G. Adlercreutz H. Prospective study of plasma enterolactone and prostate cancer risk (Sweden). Cancer Causes Control. 2004;15:1095-1102.

304 Stattin P LS. Tenkanen L. Alfthan H. Jellum E. Hallmans G. Thoresen S. Hakulinen T. Luostarinen T. Lehtinen M. Dillner J. Stenman UH. Hakama M. High levels of circulating testosterone are not associated with increased prostate cancer risk: a pooled prospective study. Int J Cancer. 2004;108:418424. 
305 Stattin P RS. Biessy C. Stenman UH. Hallmans G. Kaaks R. High levels of circulating insulin-like growth factor-I increase prostate cancer risk: a prospective study in a population-based nonscreened cohort. J Clin Oncol. 2004;22:3104-3112.

306 Stolzenberg-Solomon RZ LP. Pollak M. Taylor PR. Virtamo J. Albanes D. Insulin-like growth factor (IGF)-1. IGF-binding protein-3. and pancreatic cancer in male smokers. Cancer Epidemiol Biomarkers Prev. 2004;13:438444.

307 Storti KL BJ. Fitzgerald SJ. Zmuda JM. Cauley JA. Kriska AM. Physical activity and decreased risk of clinical gallstone disease among postmenopausal women. Prev Med. . 2005;41(3-4):772-777.

308 St-Pierre AC CB. Dagenais GR. Mauriege P. Bernard PM. Despres JP. Lamarche B. Low-density lipoprotein subfractions and the long-term risk of ischemic heart disease in men: 13-year follow-up data from the Quebec Cardiovascular Study. Arterioscler Thromb Vasc Biol. 2005;25:553-559.

309 St-Pierre AC CB. Dagenais GR. Mauriege P. Bernard PM. Despres JP. Lamarche B. Low-density lipoprotein subfractions and the long-term risk of ischemic heart disease in men: 13-year follow-up data from the Quebec Cardiovascular Study. Arterioscler Thromb Vasc Biol. 2005;25:353-359.

310 Suzuki K IY. Wakai K. Kawado M. Hashimoto S. Toyoshima H. Kojima M. Tokudome S. Hayakawa N. Watanabe Y. Tamakoshi K. Suzuki S. Ozasa K. Tamakoshi A; Japan Collaborative Cohort Study Group. Serum oxidized lowdensity lipoprotein levels and risk of colorectal cancer: a case-control study nested in the Japan Collaborative Cohort Study. Cancer Epidemiol Biomarkers Prev. 2004;13:1781-1787.

311 Svensson AM MD. Abrahamsson P. Dellborg M. Association between hyperand hypoglycaemia and 2 year all-cause mortality risk in diabetic patients with acute coronary events. Eur Heart J. 2005;26:1255-1261.

312 Sweeney C BC. Anderson KE. Lazovich D. Folsom AR. Risk factors for breast cancer in elderly women. Am J Epidemiol. 2004;160:868-875.

313 Takebayashi T NY. Uemura T. Nakashima H. Nomiyama T. Sakurai H. Omae $\mathrm{K}$. A six year follow up study of the subclinical effects of carbon disulphide exposure on the cardiovascular system. Occup Environ Med. 2004;61:127134.

314 Tan ZS SS. Beiser A. Zhang Y. Felson D. Hannan MT. Au R. Wolf PA. Kiel DP. Bone mineral density and the risk of Alzheimer disease. Arch Neurol. 2005;62:107-111.

315 Tanne D MJ. Goldbourt U. Body fat distribution and long-term risk of stroke mortality. Stroke. 2005;35:1021-1025.

316 Tarnow L AA. Parving HH. Elevated placental growth factor (PlGF) predicts cardiovascular morbidity and mortality in type 1 diabetic patients with diabetic nephropathy. Scand J Clin Lab Invest Suppl. 2005;240:73-79.

317 Tarnow L HP. Hansen BV. Borch-Johnsen K. Parving HH. Plasma N-terminal pro-brain natriuretic peptide as an independent predictor of mortality in diabetic nephropathy. Diabetologia. 2005;48:149-155.

318 Taylor EN SM. Curhan GC. Dietary factors and the risk of incident kidney stones in men: new insights after 14 years of follow-up. J Am Soc Nephrol. 2004;15:3225-3232.

319 Taylor EN SM. Curhan GC. Fatty acid intake and incident nephrolithiasis. Am J Kidney Dis. 2005;45:267-274. 
320 Taylor HA Jr CB. Garrison RJ. Andrew ME. Han H. Fox ER. Arnett DK. Samdarshi T. Jones DW. Relation of aortic valve sclerosis to risk of coronary heart disease in African-Americans. Am J Cardiol. 2005;95:401-404.

321 Thomson CC FL. Winickoff JP. Colditz GA. Camargo CA Jr. King C 3rd. Frazier AL. State tobacco excise taxes and adolescent smoking behaviors in the United States. J Public Health Manag Pract. 2004;10:490-496.

322 Thorand B KH. Baumert J. Koenig W. Chambless L. Meisinger C. Illig T. Martin S. Herder C. Elevated levels of interleukin-18 predict the development of type 2 diabetes: results from the MONICA/KORA Augsburg Study. 19842002. Diabetes. 2005;54:2392-2398.

323 Tolmunen T HJ. Ruusunen A. Voutilainen S. Tanskanen A. Valkonen VP. Viinamaki H. Kaplan GA. Salonen JT. Dietary folate and the risk of depression in Finnish middle-aged men. A prospective follow-up study. Psychother Psychosom. 2004;73:334-339.

324 Tolmunen T HJ. Voutilainen S. Ruusunen A. Alfthan G. Nyyssonen K. Viinamaki H. Kaplan GA. Salonen JT. Association between depressive symptoms and serum concentrations of homocysteine in men: a population study. Am J Clin Nutr. 2004;80:1574-1578.

325 Touvier M KE. Clavel-Chapelon F. Boutron-Ruault MC. Dual Association of beta-carotene with risk of tobacco-related cancers in a cohort of French women. J Natl Cancer Inst. 2005;97:1338-1344.

326 Tripepi G MF. Zoccali C. Inflammation markers. adhesion molecules. and allcause and cardiovascular mortality in patients with ESRD: searching for the best risk marker by multivariate modeling. J Am Soc Nephrol. 2005;16:S9398.

327 Tsai CJ. Leitzmann MF. Willett WC. Giovannucci EL. Long-term intake of dietary fiber and decreased risk of cholecystectomy in women. Am J Gastroenterol. Jul 2004;99:1364-1370.

328 Tsai CJ LM. Willett WC. Giovannucci EL. The effect of long-term intake of cis unsaturated fats on the risk for gallstone disease in men: a prospective cohort study. Ann Intern Med. 2004;141:514-522.

329 Tsai CJ LM. Willett WC. Giovannucci EL. Dietary protein and the risk of cholecystectomy in a cohort of US women: the Nurses' Health Study. Am J Epidemiol. 2004;160:11-18.

330 Tsai CJ LM. Willett WC. Giovannucci EL. Glycemic load. glycemic index. and carbohydrate intake in relation to risk of cholecystectomy in women. Gastroenterology. 2005;129:105-112.

331 Tsai CJ LM. Willett WC. Giovannucci EL. Dietary carbohydrates and glycaemic load and the incidence of symptomatic gall stone disease in men. Gut. 2005;54:823-828.

332 Tsai CJ LM. Willett WC. Giovannucci EL. Long-term intake of trans-fatty acids and risk of gallstone disease in men. Arch Intern Med. 2005;165:10111015.

333 Tseng M BR. DeVellis RF. Ziegler RG. Dietary patterns and prostate cancer risk in the National Health and Nutrition Examination Survey Epidemiological Follow-up Study cohort. . Cancer Epidemiol Biomarkers Prev. . 2004;13:7177.

334 Tseng M BR. Graubard BI. Ziegler RG. Dairy. calcium. and vitamin D intakes and prostate cancer risk in the National Health and Nutrition Examination Epidemiologic Follow-up Study cohort. Am J Clin Nutr. 2005;81:1147-1154. 
335 Tsimikas S BE. Miller ER. McConnell JP. Lennon RJ. Kornman KS. Witztum JL. Berger PB. Oxidized phospholipids. Lp(a) lipoprotein. and coronary artery disease. N Engl J Med. 2005;353:46-57.

336 Tsubono Y OT. Kobayashi M. Yamamoto S. Sobue T. Tsugane S; JPHC Study Group. No association between fruit or vegetable consumption and the risk of colorectal cancer in Japan. Br J Cancer. 2005;92:1782-1784.

337 Tsushima M NA. Lee J. Stemmermann GN. Prospective study of the association of serum triglyceride and glucose with colorectal cancer. Dig Dis Sci. 2005;50:499-505.

338 Turner BJ WM. Yang C. TenHave T. Predicting adherence to colonoscopy or flexible sigmoidoscopy on the basis of physician appointment-keeping behavior. Ann Intern Med. 2004;140:528-532.

339 Tworoger SS EA. Rosner B. Sluss P. Hankinson SE. Plasma prolactin concentrations and risk of postmenopausal breast cancer. Cancer Res. 2004;64:6814-6819.

340 Tworoger SS MS. Barbieri RL. Willett WC. Colditz GA. Hankinson SE. Plasma sex hormone concentrations and subsequent risk of breast cancer among women using postmenopausal hormones. J Natl Cancer Inst. 2005;97:595-602.

341 Ulvik A VS. Hansen S. Gislefoss R. Jellum E. Ueland PM. Colorectal cancer and the methylenetetrahydrofolate reductase $677 \mathrm{C}->\mathrm{T}$ and methionine synthase $2756 \mathrm{~A}$-> G polymorphisms: a study of 2.168 case-control pairs from the JANUS cohort. Cancer Epidemiol Biomarkers Prev. 2004;13:2175-2180.

342 Van Den Brink CL PH. Van Den Bos GA. Giampaoli S. Nissinen A. Kromhout D. Duration and intensity of physical activity and disability among European elderly men. Disabil Rehabil. 2005;27:341-347.

343 van der A DL GD. Roest M. Marx JJ. Voorbij HA. van der Schouw YT. Serum ferritin is a risk factor for stroke in postmenopausal women. Stroke. 2005;36:1637-1641.

344 van Gelder BM TM. Kalmijn S. Giampaoli S. Nissinen A. Kromhout D. Physical activity in relation to cognitive decline in elderly men: the FINE Study. Neurology. 2004;63: 2316-21.

345 van Gils CH PP. Bueno-de-Mesquita HB. Boshuizen HC. Lahmann PH. Clavel-Chapelon F. Thiebaut A. Kesse E. Sieri S. Palli D. Tumino R. Panico S. Vineis P. Gonzalez CA. Ardanaz E. Sanchez MJ. Amiano P. Navarro C. Quiros JR. Key TJ. Allen N. Khaw KT. Bingham SA. Psaltopoulou T. Koliva M. Trichopoulou A. Nagel G. Linseisen J. Boeing H. Berglund G. Wirfalt E. Hallmans G. Lenner P. Overvad K. Tjonneland A. Olsen A. Lund E. Engeset D. Alsaker E. Norat T. Kaaks R. Slimani N. Riboli E. Consumption of vegetables and fruits and risk of breast cancer. JAMA. 2005;293:183-193.

346 Van Guelpen B HJ. Johansson I. Stegmayr B. Hallmans G. Nilsson TK. Weinehall L. Witthoft C. Palmqvist R. Winkvist A. Folate. vitamin B12. and risk of ischemic and hemorrhagic stroke: a prospective. nested case-referent study of plasma concentrations and dietary intake. Stroke. 2005;36:1426-1431.

347 van Hecke MV DJ. Nijpels G. Moll AC. Heine RJ. Bouter LM. Polak BC. Stehouwer CD. Inflammation and endothelial dysfunction are associated with retinopathy: the Hoorn Study. Diabetologia. 2005;48:1300-1306.

348 van Meurs JB D-RR. Pluijm SM. van der Klift M. de Jonge R. Lindemans J. de Groot LC. Hofman A. Witteman JC. van Leeuwen JP. Breteler MM. Lips 
P. Pols HA. Uitterlinden AG. Homocysteine levels and the risk of osteoporotic fracture. N Engl J Med. 2004;350:2033-2041.

349 Verma A. Kilicaslan F. Martin DO. et al. Preimplantation B-type natriuretic peptide concentration is an independent predictor of future appropriate implantable defibrillator therapies. Heart. Feb 2006;92:190-195.

350 Visser M GB. Kritchevsky SB. Newman AB. Nevitt M. Rubin SM. Simonsick EM. Harris TB. Muscle mass. muscle strength. and muscle fat infiltration as predictors of incident mobility limitations in well-functioning older persons. $J$ Gerontol A Biol Sci Med Sci. 2005;60:324-333.

351 Voutilainen S VJ. Rissanen TH. Alfthan G. Laukkanen J. Nyyssonen K. Mursu J. Valkonen VP. Tuomainen TP. Kaplan GA. Salonen JT. Serum folate and homocysteine and the incidence of acute coronary events: the Kuopio Ischaemic Heart Disease Risk Factor Study. Am J Clin Nutr. 2004;80:317-323.

352 Vrbova L MM. Moineddin R. Jaakimainen L. Upshur RE. Does socioeconomic status affect mortality subsequent to hospital admission for community acquired pneumonia among older persons? J Negat Results Biomed. 2005;4:4.

353 Vriesendorp TM MQ. Devries JH. Legemate DA. Hoekstra JB. Early postoperative glucose levels are an independent risk factor for infection after peripheral vascular surgery. A retrospective study. Eur J Vasc Endovasc Surg. 2004;28:520-525.

354 Wakai K SK. Ito Y. Kojima M. Tamakoshi K. Watanabe Y. Toyoshima H. Hayakawa N. Hashimoto S. Tokudome S. Suzuki S. Kawado M. Ozasa K. Tamakoshi A; Japan Collaborative Cohort Study Group. Serum carotenoids. retinol. and tocopherols. and colorectal cancer risk in a Japanese cohort: effect modification by sex for carotenoids. Nutr Cancer. 2005;51:13-24.

355 Wakai K TK. Date C. Fukui M. Suzuki S. Lin Y. Niwa Y. Nishio K. Yatsuya H. Kondo T. Tokudome S. Yamamoto A. Toyoshima H. Tamakoshi A; JACC Study Group. Dietary intakes of fat and fatty acids and risk of breast cancer: a prospective study in Japan. Cancer Sci. 2005;96:590-599.

356 Wang AY SM. Tang N. Sanderson JE. Lui SF. Li PK. Woo J. Resting energy expenditure and subsequent mortality risk in peritoneal dialysis patients. J Am Soc Nephrol. 2004;15:3134-3143.

357 Wang J. Work stress as a risk factor for major depressive episode(s). Psychol Med. 2005;35:865-871.

358 Wang TJ EJ. Meigs JB. Rifai N. Fox CS. D'Agostino RB. Levy D. Vasan RS. Low-grade albuminuria and the risks of hypertension and blood pressure progression. Circulation. 2005;111:1370-1376.

359 Wang Y RE. Stampfer MJ. Willett WC. Hu FB. Comparison of abdominal adiposity and overall obesity in predicting risk of type 2 diabetes among men. Am J Clin Nutr. 2005;81:555-563.

360 Wannamethee SG LD. Whincup PH. Walker M. Ebrahim S. Davey-Smith G. Birthweight of offspring and paternal insulin resistance and paternal diabetes in late adulthood: cross sectional survey. Diabetologia. 2004;47:12-18.

361 Wark PA WM. van 't Veer P. van Wijhe G. Luchtenborg M. van Muijen GN. de Goeij AF. Goldbohm RA. van den Brandt PA. Fruits. vegetables. and hMLH1 protein-deficient and -proficient colon cancer: The Netherlands cohort study. Cancer Epidemiol Biomarkers Prev. 2005;14:1619-1625.

362 Wattanakit K FA. Selvin E. Weatherley BD. Pankow JS. Brancati FL. Hirsch AT. Risk factors for peripheral arterial disease incidence in persons with 
diabetes: the Atherosclerosis Risk in Communities (ARIC) Study.

Atherosclerosis. 2005;180:389-397.

363 Weinstein AR SH. Lee IM. Cook NR. Manson JE. Buring JE. Gaziano JM. Relationship of physical activity vs body mass index with type 2 diabetes in women. JAMA. 2004;292:1188-1194.

364 Weinstein SJ. Wright ME. Pietinen P. et al. Serum alpha-tocopherol and gamma-tocopherol in relation to prostate cancer risk in a prospective study. $J$ Natl Cancer Inst. Mar 2 2005;97:396-399.

365 Whittemore AS CP. Feldman D. Cohn BA. Prostate specific antigen levels in young adulthood predict prostate cancer risk: results from a cohort of Black and White Americans. J Urol. 2005;174:872-876.

366 Wolf M SJ. Shah A. Vossen Smirnakis K. Jimenez-Kimble R. Ecker JL. Thadhani R. Inflammation and glucose intolerance: a prospective study of gestational diabetes mellitus. Diabetes Care. 2004;27:21-27.

367 Wolk R BP. Lennon RJ. Brilakis ES. Johnson BD. Somers VK. Plasma leptin and prognosis in patients with established coronary atherosclerosis. $J$ Am Coll Cardiol. 2004;44:1819-1824.

368 Wong TY KM. Klein R. Klein BE. Hubbard LD. A prospective cohort study of retinal arteriolar narrowing and mortality. Am J Epidemiol. 2004;159:819825.

369 Wong TY KR. Sharrett AR. Duncan BB. Couper DJ. Klein BE. Hubbard LD. Nieto FJ; Atherosclerosis Risk in Communities Study. Retinal arteriolar diameter and risk for hypertension. Ann Intern Med. 2004;140:248-255.

370 Wong TY SA. Klein R. Klein BE. Retinal vessel diameters and the incidence of gross proteinuria and renal insufficiency in people with type 1 diabetes. Diabetes. 2004;53:179-184.

371 Wong TY SA. Klein R. Klein BE. Hubbard LD. Retinal arteriolar narrowing. hypertension. and subsequent risk of diabetes mellitus. Arch Intern Med. 2005;165:1060-1065.

372 Wrensch M WA. Wiencke J. Miike R. Sison J. Wiemels J. Barger G. DeLorenze G. Aldape K. Kelsey K. History of chickenpox and shingles and prevalence of antibodies to varicella-zoster virus and three other herpesviruses among adults with glioma and controls. Am J Epidemiol. 2005;161:929-938.

373 Wright ME MD. Pietinen P. Taylor PR. Virtamo J. Albanes D. Estimated Urine $\mathrm{pH}$ and Bladder Cancer Risk in a Cohort of Male Smokers (Finland)*. Cancer Causes Control. 2005;16:1117-1123.

374 Wright ME MS. Stolzenberg-Solomon RZ. Li Z. Pietinen P. Taylor PR. Virtamo J. Albanes D. Development of a comprehensive dietary antioxidant index and application to lung cancer risk in a cohort of male smokers. Am J Epidemiol. 2004;160:68-76.

375 Wu K HF. Fuchs C. Rimm EB. Willett WC. Giovannucci E. Dietary patterns and risk of colon cancer and adenoma in a cohort of men (United States). Cancer Causes Control. 2004;15:853-862.

376 Yamagata H KY. Nakamura S. Kubo M. Tanizaki Y. Matsumoto T. Tanaka K. Kato I. Shirota T. Iida M. mpact of fasting plasma glucose levels on gastric cancer incidence in a general Japanese population: the Hisayama study. Diabetes Care. 2005;28:789-794.

377 Yang G SX. Jin F. Elasy T. Li HL. Li Q. Huang F. Zhang XL. Gao YT. Zheng W.Yang G. Shu XO. Jin F. Elasy T. Li HL. Li Q. Huang F. Zhang XL. Gao YT. Zheng W. Soyfood consumption and risk of glycosuria: a cross-sectional 
study within the Shanghai Women's Health Study. Eur J Clin Nutr. 2004;58:615-620.

378 Yeh HC PN. Wang NY. Pankow JS. Duncan BB. Brancati FL. Vital capacity as a predictor of incident type 2 diabetes: the Atherosclerosis Risk in Communities study. Diabetes Care. 2005;28:1472-1479.

379 Yende S. Tuomanen EI. Wunderink R. et al. Preinfection systemic inflammatory markers and risk of hospitalization due to pneumonia. Am J Respir Crit Care Med. Dec 1 2005;172:1440-1446.

380 Yuan JM RR. Gao YT. Qu YH. Chu XD. Yu MC. Prediagnostic levels of serum micronutrients in relation to risk of gastric cancer in Shanghai. China. Cancer Epidemiol Biomarkers Prev. 2004;13:1772-1780.

381 Zeleniuch-Jacquotte A AH. Shore RE. Koenig KL. Kato I. Arslan AA. Toniolo P. Circulating enterolactone and risk of breast cancer: a prospective study in New York. Br J Cancer. 2004;91:99-105.

382 Zeleniuch-Jacquotte A GY. Shore RE. Koenig KL. Arslan AA. Kato I. Rinaldi S. Kaaks R. Toniolo P. Postmenopausal levels of sex hormones and risk of breast carcinoma in situ: results of a prospective study. Int J Cancer. . 2004;114:323-327.

383 Zeleniuch-Jacquotte A SR. Koenig KL. Akhmedkhanov A. Afanasyeva Y. Kato I. Kim MY. Rinaldi S. Kaaks R. Toniolo P. Postmenopausal levels of oestrogen. androgen. and SHBG and breast cancer: long-term results of a prospective study. Br J Cancer. 2004;90:153-159.

384 Zhang C WM. Sorensen TK. King IB. Kestin MM. Thompson ML. Leisenring WM. Dashow EE. Luthy DA. Maternal plasma ascorbic Acid (vitamin C) and risk of gestational diabetes mellitus. Epidemiology. 2004;15:597-604.

385 Zhang C WM. Frederick IO. King IB. Sorensen TK. Kestin MM. Dashow EE. Luthy DA. Vitamin C and the risk of gestational diabetes mellitus: a casecontrol study. J Reprod Med. 2004;49:257-266.

386 Zhang SM HS. Hunter DJ. Giovannucci EL. Colditz GA. Willett WC. Folate intake and risk of breast cancer characterized by hormone receptor status. Cancer Epidemiol Biomarkers Prev. 2005;14:2004-2008.

387 Zhang X SX. Gao YT. Yang G. Matthews CE. Li Q. Li H. Jin F. Zheng W. Anthropometric predictors of coronary heart disease in Chinese women. Int $J$ Obes Relat Metab Disord. 2004;28:734-740.

388 Zhang X SX. Li H. Yang G. Li Q. Gao YT. Zheng W. Prospective cohort study of soy food consumption and risk of bone fracture among postmenopausal women. Arch Intern Med. 2005;165:1890-1895.

389 Zylberstein DE BC. Bjorkelund C. Landaas S. Sundh V. Thelle D. Lissner L. Serum homocysteine in relation to mortality and morbidity from coronary heart disease: a 24-year follow-up of the population study of women in Gothenburg. Circulation. 2004;109:601-606. 